\title{
The detection of the motion of contrast modulation: A parametric study
}

\author{
Simon J. Cropper and Jessica G. S. KvansakUl \\ University of Melbourne, Melbourne, Victoria, Australia \\ AND \\ Alan Johnston \\ University College London, London, England
}

\begin{abstract}
Despite a long and productive history as a focus of research interest, the details of how humans detect motion in an image remain controversial. This debate has not been helped by the lack of a clear parametric description of motion discrimination for some of the more simple visual stimuli employed in the literature to date. With this in mind, in the present work, we examined a peculiarity observed in the perception of the motion of second-order (contrast-modulated) stimuli: Under certain stimulus conditions, there is a reversal in the perceived direction of motion of the pattern. The aim was to quantify this phenomenon, relate the reversal to forward (veridical) and ambiguous motion, and place the behavioral data in the context of the window of visibility model of spatiotemporal contrast sensitivity. The direction of motion of contrast-modulated patterns was measured as a function of temporal frequency and carrier contrast, under different critical stimulus conditions. The stimulus properties manipulated were spatial frequency, spatial-phase relationship of carrier and sidebands, color, duration, and, most critically, the retinal location of the stimulus. On a purely empirical basis, the data reconciled several conflicts in the recent literature. From a theoretical standpoint, the data were well explained by the window of visibility approach in the majority of conditions and were partially explained in the remaining conditions. The results raise some interesting questions about underlying motion detection mechanisms and the assumptions embodied in our approach to motion modeling and the visual system in general. Supplemental materials for this article may be downloaded from app.psychonomic-journals.org/content/supplemental.
\end{abstract}

The ability to accurately identify motion in an image is a critical property of the visual system, and one that has attracted a great deal of research interest over the past 30 years or so. However, despite the wealth of data collected and the extent of the confluence of that data, there is still uncertainty regarding how we detect the motion of the simplest luminance edge, let alone the more complex patterns employed in much of the recent research in motion psychophysics. It certainly appears that the motion detection system is a strongly hierarchical process and that the initial signal specific to the motion subsystem is related to the direction of motion of an edge (Lennie \& Movshon, 2005; Marr, 1982). The edge is usually coded by a firstorder modulation of the image statistics (Cavanagh \& Mather, 1989; Julesz, 1971), and the directional signal relating to any 1 -D edge is accurate only to within $\pm 90^{\circ}$, a property known as the aperture problem (Marr \& Ullman, 1981). From this relatively simple starting point, the signal is progressively built into a neural representation that is a remarkably complex and powerful contributor to the overall percept of the visual scene, revealing not only the motion in the input, but also the depth and, in some cases, the form (Warren, 2004). Furthermore, visual motion in an image is strongly linked to the change in apparent position of a perceptually consistent auditory signal, indicating its importance as a key signal facilitating the integration of the internal representation of the external environment as a whole (e.g., Kitagawa \& Ichigara, 2002). The hierarchy of signal construction is seen in both the behavioral data (Wilson, Ferrera, \& Yo, 1992) and the neurophysiological data (Duffy, 2004), evidence that, in turn, shapes the way in which we define and describe the stimuli that we use to further explore the issue.

An example of the influence of the apparent hierarchy is the description of a visual stimulus in terms of its firstorder and second-order spatial statistics and of the consideration of each spatial dimension ( $x$ and $y$ ) independently. Consistent with this, there has been an argument presented within the motion literature for independent pathways in the system that deal with the first-order and second-order components of the pattern separately (e.g., Badcock \& Derrington, 1985; Badcock \& Khuu, 2001; Edwards \& Badcock, 1995; Ledgeway \& Smith, 1994b; Lu \& Sperling, 1995, 2001; Wilson et al., 1992). Furthermore, it has been thought that the early cortical pathways are principally spatially $1-D$ in their sensitivity, a 2-D percept being 
recovered from those 1-D components, as is the overall spatial structure.

These early stages of the visual pathway, which deal with the transduction and translation of the atomic components of the visual image, fragmented at the level of the retina and constructed into a meaningful internal representation thereafter, place a constraint of visibility upon all subsequent processing of the image. Put most simply, if the initial stages of the system cannot detect an image component at the outset, it cannot contribute to the resultant neural image. This has been formalized in a model of visual sensitivity known as the window of visibility (Watson, Ahumada, \& Farrell, 1986), which relates the stimulus's spatiotemporal properties to the system's ability to detect and discriminate them. We have used this framework with significant success in previous work to explain the ability to discriminate sampled motion sequences in different stimuli (Cropper \& Badcock, 1994), and we again utilize this model of early limits on visual perception in the analysis of the data presented below. That is not to say that the window of visibility explains how we see motion in second-order stimuli; it has no directional selectivity as a stand-alone mechanism and simply modulates the inputs to any subsequent processing. What we are interested in thoroughly testing is whether these constraints will explain a range of directional percepts (forward, ambiguous, reversed) across a multidimensional parameter space.

\section{Specifics}

The work described in this article was concerned with one of the most simple and early components of the composite motion structure in the neural representation of the image: a second-order spatial modulation in a single spatial dimension. Specifically, in the work described here, we examined some peculiarities observed in the detection of the motion of the contrast variation of a one-dimensional carrier grating. These stimuli are uniquely defined by reference to their second-order spatial statistics (Cavanagh $\&$ Mather, 1989; Julesz, 1971) and allow examination of the most rudimentary processes involved in extracting the contrast envelope of a carrier pattern and discriminating its direction of motion. The second-order stimulus category also relates to nonlinear (Boulton \& Baker, 1993) or non-Fourier (Chubb \& Sperling, 1988) motion processing, although the description itself is dependent only on the spatial, rather than spatiotemporal, statistics (Cropper \& Johnston, 2001). The process-based definitions pertaining to these patterns arise from the proposal that a nonlinear process, other than the actual directional segregation, must be implemented in order to detect the motion of a property of the stimulus that is not uniquely specified by a component in the Fourier representation of the image.

It has been argued that the simplest form of the class of patterns referred to as second-order is the modulation of the contrast of a carrier grating (Cropper, 1998), and much of the earlier work with these types of patterns employed these quite simple stimuli (Badcock \& Derrington, 1985, 1987, 1989; Benton \& Johnston, 2001; Benton, Johnston, \& McOwan, 1997; Cropper, 1994, 1998; Cropper \& Derrington, 1994, 1996; Derrington, Badcock, \& Henning,
1993; Derrington \& Henning, 1987, 1993; Henning, Hertz, \& Broadbent, 1975; Holliday \& Anderson, 1994; Jamar, Campagne, \& Koenderink, 1982; Jamar, Kwakman, \& Koenderink, 1984; Johnston \& Benton, 1997; Johnston, Benton, \& McOwan, 1999; Johnston \& Clifford, 1995; Lombrozo, Judson, \& MacLeod, 2005). A more complex version based on similar principles, where the carrier is a noise field rather than a grating (Chubb \& Sperling, 1988), has also been used extensively in the literature (Benton \& Johnston, 1997; Ledgeway \& Hess, 2000, 2002; Ledgeway, Hess, \& McGraw, 2002; Ledgeway \& Hutchinson, 2005, 2006; Ledgeway \& Smith, 1994a, 1994b, 1995; Lu \& Sperling, 1995, 2001; Smith \& Ledgeway, 1997, 1998, 2001). To a large degree, it was assumed for some time, both implicitly and explicitly, that the carrier form was unimportant in dictating the ability to discriminate the motion of the second-order contrast envelope, but this has recently been shown to be unjustified, suggesting the need for a revaluation of the data and their implications. For examination of the differences between narrow-band and noise, 1-D and 2-D carriers, the reader is referred elsewhere (Cropper \& Badcock, 1995, 2008; Cropper \& Johnston, 2001; Smith \& Ledgeway, 1995). ${ }^{1}$

Furthermore, recent reviews (Derrington, Allen, \& Delicato, 2004; Lu \& Sperling, 2001) have underscored the bottom line, which is that although there is a wealth of data on our ability to detect and discriminate motion in a contrast modulation, we still do not know how it is achieved neurally or mechanistically, and there is not even broad agreement on the basic properties of the ability, since it appears to be so critically linked to the first-order properties of the stimulus. In an attempt to partially address this problem, the present article takes a parametric approach to a particular aspect of the detection of the motion of a contrast envelope and relates the properties of the simplest of carriers to the ability to discriminate the motion of a contrast-modulating envelope.

Differences in the ability of observers to discriminate the direction of motion of first- and second-order patterns have been shown at both low and high temporal drift frequencies, and these differences in performance are often taken as evidence for the existence of different mechanisms (or pathways) for the detection of motion of the two types of stimuli. This may indeed be considered to be the most intuitive interpretation of such data on one level and forms the argument for the parallel structure of one class of model developed to explain motion detection in these stimuli (Lu \& Sperling, 1995; Wilson et al., 1992).

Examples of performance differences between firstand second-order stimuli can be seen at both ends of the temporal frequency range of discriminable motion. The lower temporal frequency limit for discrimination of the direction of motion is generally found to be higher for second-order stimuli. This means that for a secondorder modulation, one requires a greater temporal drift frequency for an observer to discriminate its direction of motion than for an equivalent luminance modulation. On the other hand, however, there have been reports of similar performance for first- and second-order stimuli in other motion tasks (Ledgeway \& Smith, 1997; Lu \& Sperling, 
1995; Smith, Hess, \& Baker, 1994), leading to difficulties in drawing general conclusions about underlying mechanisms on the basis of differences in task performance for luminance-defined and contrast-defined stimuli.

At the opposite range of discriminable rates of motion, the upper temporal limit for direction discrimination has been a scalar that has led to even greater disagreement in recent literature, and it is with this measure that the present article is most concerned. Again, the upper temporal resolution of observers' ability to discriminate motion in second-order stimuli is generally thought to be compromised, when compared with that for luminance (first-order) stimuli. There are several reports that when contrast-modulated grating patterns drift faster than around $4-6 \mathrm{~Hz}$, observers find it very difficult to discriminate their direction of motion (Cropper, 1992, 1994; Cropper \& Derrington, 1994, 1996; Derrington et al., 1993). These data appear to be contradicted by reports that observers can discriminate the motion of a rapidly moving $(>10 \mathrm{~Hz}) 100 \%$ contrast-modulated grating (a beat) if the carrier contrast is sufficiently high (Holliday \& Anderson, 1994). The most intuitive explanation for the difference in the reported data (for central vision) is that the increased carrier contrast introduces a visible nonlinear component in the stimulus at the envelope frequency that can then be detected by the motion system (Burton, 1973; Henning et al., 1975; Holliday \& Anderson, 1994; MacLeod, Williams, \& Makous, 1992).

A critical issue when first-order and second-order stimuli are compared is to establish some equality between the two types of modulation. One of the more intuitive methods is to scale the stimuli to the observer's ability to discriminate the modulation in question from an unmodulated carrier and define this as the envelope detection threshold or, more correctly, the discrimination threshold. When luminance and contrast modulation are compared, the luminance is compared against a blank field, whereas the contrast is compared against its unmodulated luminance-coded carrier. Even this measure is not as straightforward as it may seem (Cropper, 1998). This discrimination threshold remains, however, the most convenient way of achieving some equivalence between the different stimuli, and this method will be adopted in the present work. First-order (carrier) and second-order (envelope) stimulus modulation will also be used as an independent variable in order to overcome any serious issues with regard to this choice of scale factor.

An anomalous aspect of observers' performance that is of particular interest in the present work is that there is occasionally a reversal in the perceived direction of motion of contrast-modulated gratings as their temporal frequency increases (Derrington et al., 1993). This has been explained on the basis of the relative visibility of the two sideband Fourier components of the pattern (see Equation 4 in the online supplemental material) - specifically, that the lower frequency sideband becomes relatively more visible as temporal frequency increases. This component moves in the opposite direction to the envelope and is thought to underlie the apparent reversal of direction. This approach is consistent with the window of visibility theoretical framework (Watson et al., 1986) introduced above, and the model has been exploited, in the spatial domain, to account for the falloff in directionally specific sensitivity to the contrast modulation as stimuli move into the periphery (Wang, Hess, \& Baker, 1997). Although the window of visibility explicitly affects all subsequent processing, it is the imbalance introduced into the component representation of the stimulus by the window that is suggested to cause the reversal in apparent motion of an otherwise driftbalanced stimulus (Chubb \& Sperling, 1988).

The work described in this article parametrically quantifies this perceived reversal in both the spatial and the temporal domains and places it in the context of the more commonly observed inability to discriminate any consistent directional motion. The window of visibility theory is taken as the default explanation and is evaluated against the data, an approach that has served us well in the past (Cropper \& Badcock, 1994). We then consider what aspects of the data may be accounted for by the window of visibility framework and whether a more elaborate theory is required. It is worth noting that any more complex theory must also incorporate the physical limits on motion processing embodied in the window of visibility approach to visual processing. By this means, we hope to address the question of whether there is a need to invoke separate pathways in the motion detection system in order to explain differences in the pattern of responses for first- and second-order stimuli and perhaps provide some clarity on the relationship between the extensive behavioral data on data motion detection and the theoretical literature. A specific comparison of the existing models has been presented in an accompanying article (Cropper, Johnston, \& Benton, 2008).

\section{METHOD}

\section{Apparatus and Stimuli}

All patterns were digitally generated from sinusoidal modulations of color or luminance and were displayed to a contrast resolution of 14 bits by a VSG2/3 (Cambridge Research Systems) stimulus generator. The patterns were presented on a Mitsubishi HL7955 color monitor with a mean luminance of $44.2 \mathrm{~cd} / \mathrm{m}^{2}$ and chromaticity at CIE coordinates of $x=0.333, y=0.377$, or on a Sony SEII monitor of the same mean luminance and chromaticity. The monitors were driven at a frame rate of $120 \mathrm{~Hz}$ and a line rate of $75 \mathrm{kHz}$, with all patterns created digitally before presentation. The voltage-toluminance relationship of the display was measured using a photometric head (Graseby S351G), and the nonlinear relationship was corrected using internal lookup tables on the VSG. The curve-fitting procedure gave an $R$ value accounting for .998 of the variance. The display subtended a visual angle of $30^{\circ} \times 24^{\circ}$ at the viewing distance of $0.5 \mathrm{~m}$, and the stimuli occupied a portion of this total display area. The visible stimulus dimensions depended on the experiment in question but were selected from a $20^{\circ}$ disk, a $6^{\circ}$ disk, a $4^{\circ}$ disk, or an annulus with a $20^{\circ}$-outer $/ 8^{\circ}$-inner diameter. Each experiment and figure detail the particular conditions used. A small dark fixation point was located at the center of the display. Viewing was conducted in a semidarkened room and was binocular with natural pupils. No head restraint was used. The observers were the author (S.J.C.) and 4 paid observers naive as to the aim of the experiment (C.L.W., L.K., R.D., and D.M.). 
Grating-Single Component

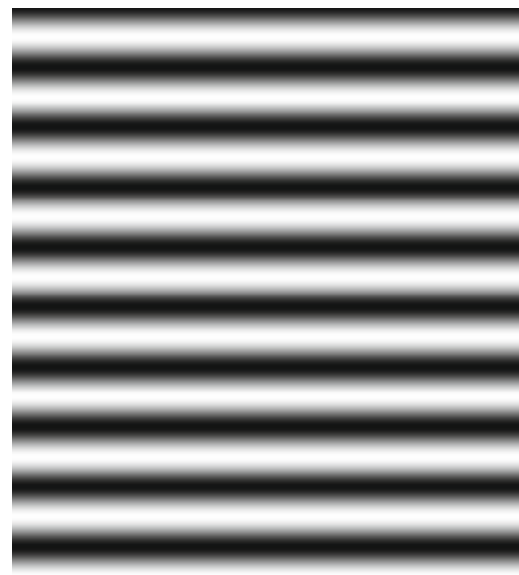

QFM-Three Components

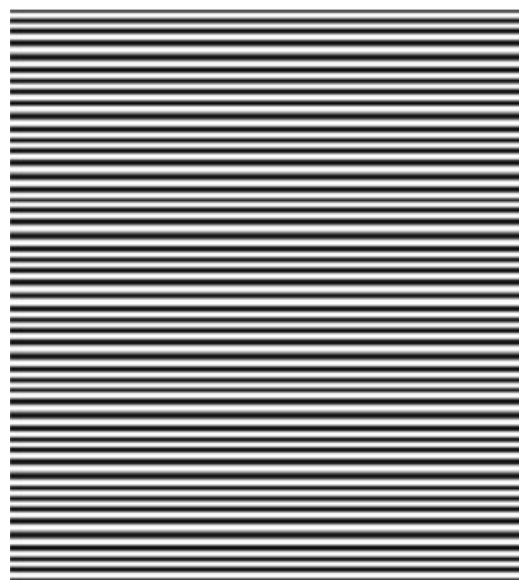

Beat-Two Components

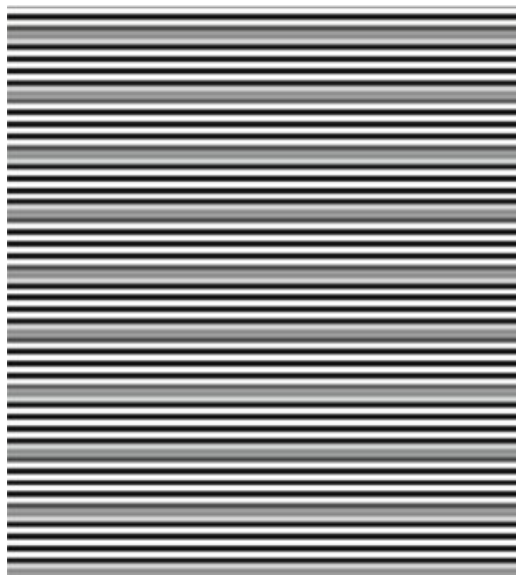

AM-Three Components

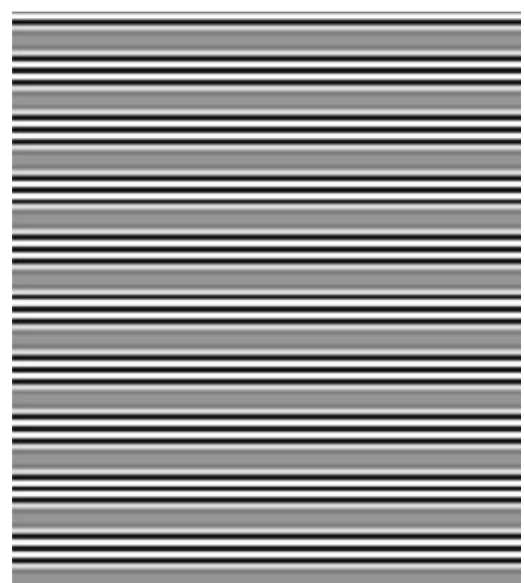

Figure 1A. The stimuli used in this study. Static representations of the four stimulus types normalized to a carrier contrast of 1 . AM, amplitude modulated; QFM, quasifrequency-modulated.

Spatiotemporal properties. The stimuli were simple sinusoidal gratings, beat patterns, amplitude-modulated (AM) or quasi-frequency-modulated (QFM) gratings. ${ }^{2}$ All patterns were 1-D, horizontal, and either achromatic modulations in luminance or subjectively equiluminant modulations of color. In an attempt to abbreviate this somewhat oversized article, equations describing the stimuli are placed in the supplemental materials available on the journal Web site. Graphical examples of the patterns are given in Figure 1A, with the luminance (or chromatic) profiles shown in Figure 1B. Figure 1C shows space-time plots of the figures for slow-moving (left-hand panel) and fast-moving (right-hand panel) stimuli, all moving upward.

In the present study, all the stimuli were restricted by a raisedcosine temporal envelope, and the duration, given in the Results section, was half the width of this envelope. The stimuli were moved smoothly and continuously to the maximum resolution of the frame rate $(75 \mathrm{~Hz})$. No frame or line interleaving was employed. If the stimulus duration was not used as an independent variable, the presentation was set at $0.5 \mathrm{sec}$.

Spatiochromatic properties. All the stimuli were described as vector excursions in a fully calibrated cardinal color space (Derrington, Krauskopf, \& Lennie, 1984). Luminance stimuli were achromatic modulations along an $\mathrm{L}+\mathrm{M}(+\mathrm{S})$ vector; chromatic mod- ulation was along an $\mathrm{L}-\mathrm{M}$ vector in a subjectively equiluminant plane for each observer. Subjective equiluminance was achieved through heterochromatic flicker photometry at temporal frequencies spanning the range used in the direction discrimination task. It has previously been shown that within this range, there is little significant variation as a function of temporal frequency at the spatial frequencies employed in this study (Cropper, 2005). All chromatic components (Experiment 2.4) were below $1.1 \mathrm{cpd}$ to minimize the intrusion of unwanted luminance artifacts.

\section{Psychophysical Methods}

Detection thresholds. The experimental paradigm used to measure detection thresholds for the simple carrier gratings was a standard two-alternative forced choice (2AFC) detection task. A stimulus was presented in one of two intervals, and the observer indicated, via a mousepress, in which interval the stimulus had appeared; a feedback tone was then provided to the observer. The contrast of the stimulus was adjusted according to the observer's response by a modified PEST staircase procedure (Findlay, 1978) that gave a final threshold estimate of performance at the level of $75 \%$ correct. To measure detection threshold for the second-order amplitude modulation, the carrier was set to a given contrast above detection threshold, and the depth of modulation was varied while the carrier contrast 


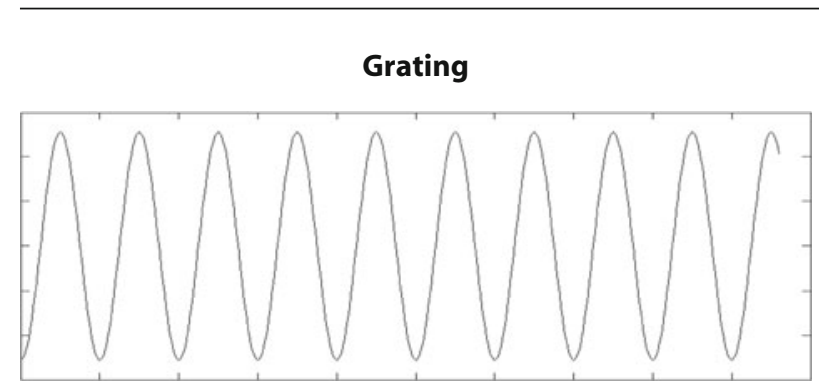

Beat

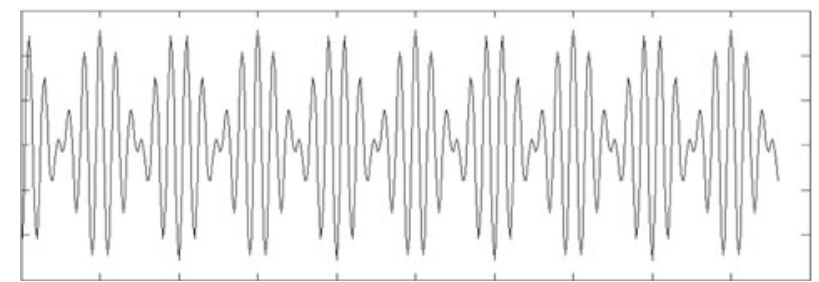

AM Grating

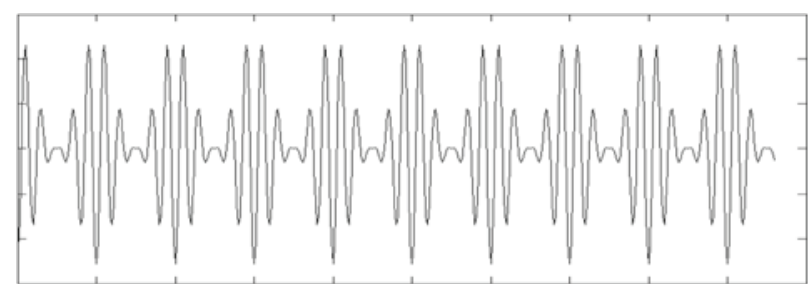

QFM Grating

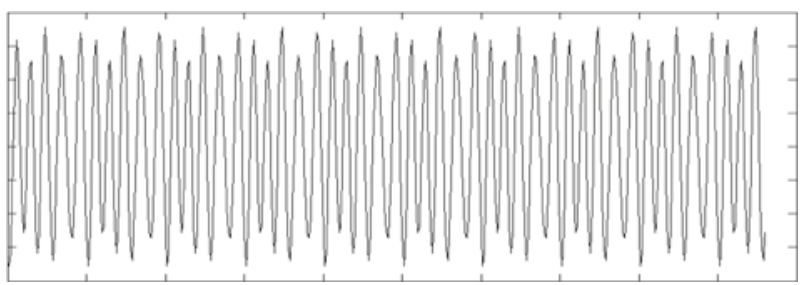

Figure 1B. Luminance profiles of each stimulus. AM, amplitude modulated; QFM, quasi-frequency-modulated.

was kept constant. The nonsignal interval contained the carrier at a contrast randomly chosen to be between the mean (carrier) contrast in the signal interval and the peak contrast in that interval to overcome the influence of any spurious contrast cue in the task, a method tested and validated in previous work (Cropper, 1998). All the stimuli were scaled to visibility for the particular conditions of presentation measured in the subsequent direction discrimination task.

Direction discrimination thresholds. A two-interval, twoalternative forced choice direction discrimination procedure, combined with the method of constant stimuli, was used to measure the observers' ability to discriminate the direction of motion of the stimuli. The observers had to indicate the interval in which the stimulus moved upward. There were two stimulus intervals in which the stimulus moved in opposite directions, randomly allocated at the start of each interval pair. No feedback was given, since there was no "correct" response.

Experimental structure. A parametric study such as this can be particularly unwieldy to digest and understand simply because of the bulk of the data and the number of parameters varied. In order to assist the reader in this, Table 1 presents a summary of each experiment, giving its purpose, the variables manipulated, the stimulus conditions, the figure number where the data is plotted, the window of visibility prediction, and whether this was met by the data or not.

\section{RESULTS}

\section{Experiment 1: Discrimination of the Direction of Motion of AM Gratings As a Function of Temporal Drift Frequency}

Experiment 1 quantifies the observers' ability to discriminate the direction of motion of an AM grating presented at a moderate carrier contrast of $1.0 \log$ units (10 times) above direction threshold and an envelope modulation depth of twice (or 3 times for observer D.M.) detection threshold, which amounts to a depth in the region of $50 \%$ and $75 \%$, respectively (Cropper, 1998). The stimulus was presented centrally in a $20^{\circ}$ circular window. Detection threshold was independently measured for each presentation duration, making all the stimuli similarly and consistently scaled to visibility. It is worth noting, however, that the ability to discriminate the envelope modulation from its carrier is largely independent of stimulus duration once the carriers are also scaled to detection threshold (Cropper, 1998). The observers were required to discriminate the direction of motion of the luminance AM grating at durations between 0.03125 and $0.5 \mathrm{sec}$ (expressed as half the width of the raised-cosine temporal envelope). These moderate conditions are relevant since they show (1) the basic ability of observers to discriminate the direction of motion of a contrast envelope, (2) the reduced temporal resolution of the mechanism involved in the discrimination task as temporal frequency increases (Derrington \& Cox, 1998; Lu \& Sperling, 1995, 2001; Smith \& Ledgeway, 1998), and (3) the reversal of perceived motion at the highest temporal frequency, which then forms the principal point of interest of the rest of the article.

Figure 2 plots the performance (proportion perceived upward) in the $2 \mathrm{AFC}$ direction discrimination task as a function of the temporal drift frequency of the envelope. The carrier always remained stationary. Data for 2 observers are shown: S.J.C. (envelope twice threshold only) and D.M. (envelope twice and three times threshold). Each data point is the result of 100 observations, and the error bars are \pm 1 SEM.

The principal feature of the data to notice is that when the presentation duration is greater than $0.125 \mathrm{sec}$ (open symbols), the curve is strongly biphasic in form. This indicates that the direction of drift was seen veridically within the region of $0.5-3 \mathrm{~Hz}$, although the precise thresholds are duration dependent; at greater temporal frequencies, the patterns were perceived as either moving ambiguously $(3-5 \mathrm{~Hz})$ or moving backward $(>5 \mathrm{~Hz})$. As has previously been shown (Cropper \& Derrington, 1994, 1996; Derrington et al., 1993), at durations shorter than around $0.0625-0.125 \mathrm{sec}$, the direction of motion (forward or backward) is not discriminable when the moving attribute of that pattern is its contrast envelope. This is supported by the flatter functions for the shorter duration stimuli (closed symbols; see also Cropper, 2005). At the 


\section{Space-Time Plots}
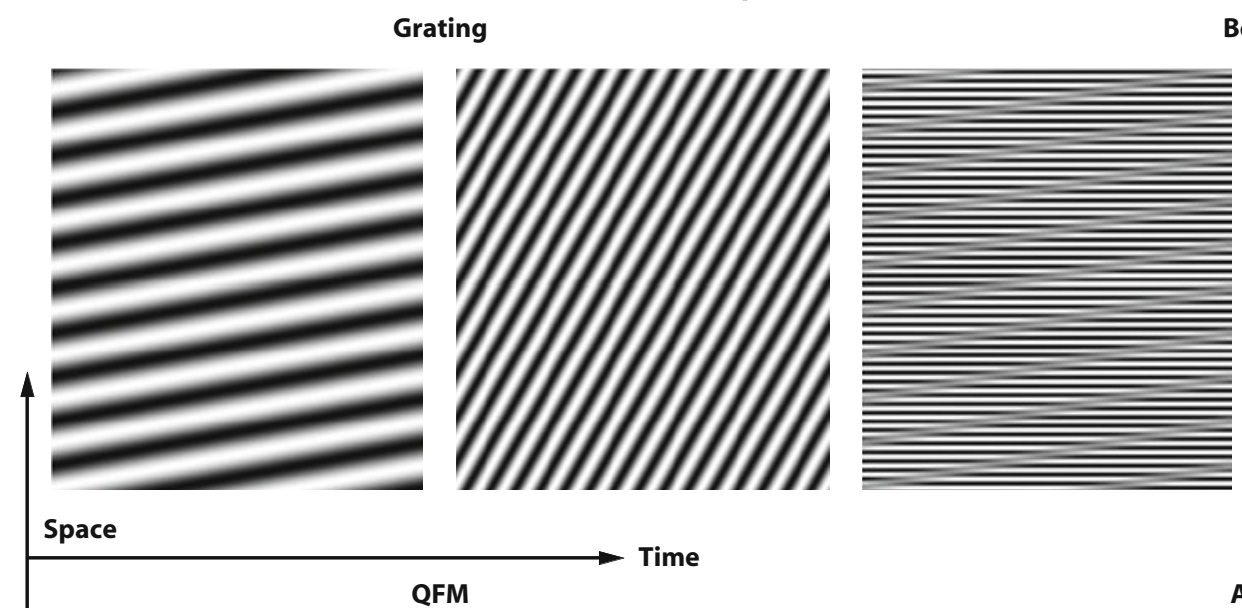

Beat

QFM
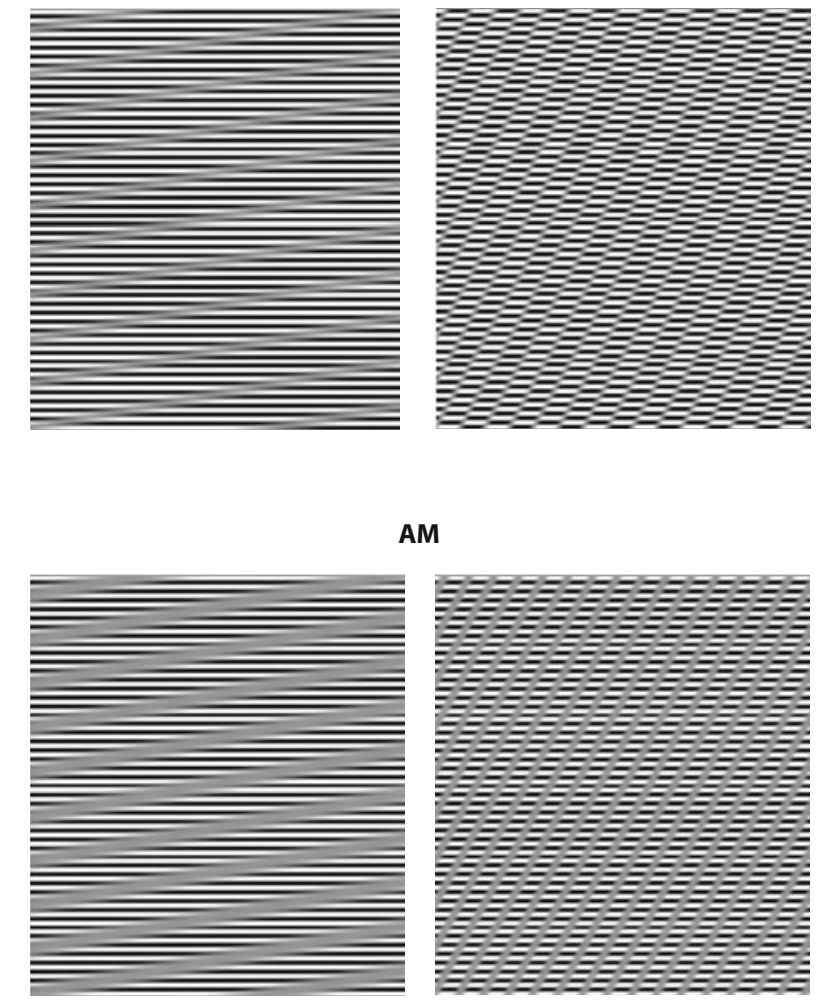

AM
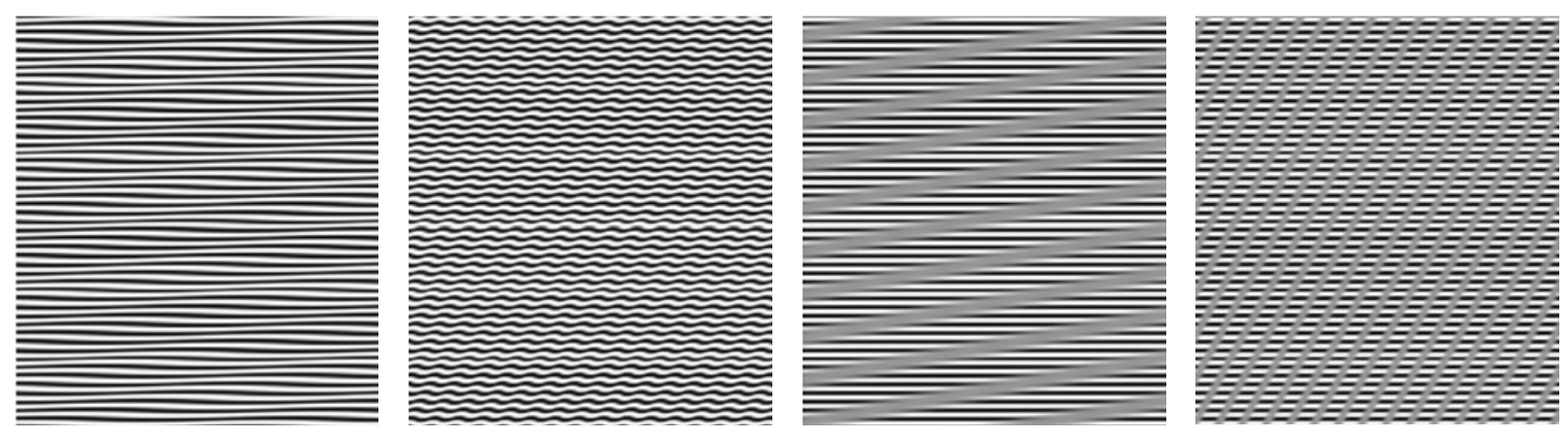

Figure 1C. Space-time plots of the drifting stimuli at slow (left-hand panel of each pair) and fast (right-hand panel of each pair) envelope drift rates. AM, amplitude modulated; QFM, quasi-frequency-modulated.

durations at which a correct direction of motion (i.e., forward) is discriminable, the temporal frequency at which peak performance occurs increases as duration decreases (Seiffert \& Cavanagh, 1998).

Even when the depth of modulation of the envelope is increased to three times discrimination threshold (around $75 \%$ for Observer D.M.; Cropper, 1998) there is little change in the pattern of results. There is slight enhancement of the biphasic function for the 0.0625 -sec-duration stimulus. The reversal of motion in a $100 \%$-modulated contrast envelope (Derrington et al., 1993; Experiment 2.2 in the present article) implies that the misperception of motion is not simply a result of a poor internal representation of the envelope, relative to the carrier. The majority of the following data ensure that the depth of modulation of the envelope and, presumably, the strength of the internally generated signal corresponding to the second-order modulation is not a limiting factor by using $100 \%$-modulated contrast envelopes. Where there is some reason to believe that this factor may be relevant to the data, the depth of envelope modulation is modified accordingly.

\section{Experiment 2: An Examination of the Basis for the Reversal: Data and Window of Visibility Predictions}

The data presented in Experiment 1 illustrate the basic effect under examination in the present article: the influ- ence of the temporal frequency upon the perceived direction of motion of a contrast envelope. The reversal seen at higher temporal frequencies appears to occur only when the carrier contrast is insufficient to generate some internal signal that can be detected by mechanisms generally specialized to deal with moving first-order (luminance) signals (Derrington, 1994; Holliday \& Anderson, 1994). In the remainder of the article, we will examine the conditions under which this reversal occurs and whether the most parsimonious - and in many cases, the defaultexplanation, the window of visibility model (Watson et al., 1986), is actually sufficient to explain the data.

Experiment 2.1: The effect of spatial frequency. The clearest prediction made by the component-based window of visibility approach to the issue at hand is based on the spatial frequency content of the stimulus. This has recently been reexamined in the spatial domain (Wang et al., 1997). The prediction is that as the components of the AM pattern move further apart in spatial frequency, the upper sideband - that is, the component with the highest spatial frequency (Equation 4) - will be comparatively less visible as its temporal frequency increases. This is due to the form of the spatiotemporal contrast sensitivity function. The resulting effect upon the appearance of the composite pattern is that the envelope spatial frequency approaches that of the carrier (assuming that the carrier remains at the same spatial frequency in all cases). Thus, 
Table 1

Outline of Each Experiment, Its Purpose and Variables, and the Simplest Prediction Made by the Window of Visibility Model

\begin{tabular}{|c|c|c|c|}
\hline Experiment & Conditions/Variables/Figure Numbers & $\begin{array}{c}\text { Window of } \\
\text { Visibility Prediction }\end{array}$ & $\begin{array}{l}\text { Prediction Met? } \\
\text { (see Discussion) }\end{array}$ \\
\hline 1 & $\begin{array}{l}\text { Baseline data showing forward and reversed motion } \\
\text { Conditions: AM; carrier, } 1 \mathrm{cpd} ; 10 \times \text { threshold; } 20^{\circ} \text { central disk; } \\
\quad \text { envelope, } 0.2 \mathrm{cpd} 2 \times, 3 \times \\
\text { Variables: Temporal drift rate }(0.4-16 \mathrm{~Hz}) \\
\text { Duration }(0.03125-0.5 \mathrm{sec}) \\
\text { Figure: } 2\end{array}$ & $\begin{array}{l}\text { Baseline conditions-increasing } \\
\text { temporal frequency elicits reversal. }\end{array}$ & yes \\
\hline 2.1 & $\begin{array}{l}\text { The effect of spatial frequency } \\
\text { Conditions: AM; carrier, } 1 \mathrm{cpd} ; 20^{\circ} \text { central disk; } 2 \text { - and } 16-\mathrm{Hz} \text { drift; } \\
\quad \text { duration, } 0.5 \mathrm{sec} \text {; envelope, } 100 \% \text { modulated } \\
\text { Variables: Carrier contrast, } 10 \times-200 \times \text { threshold } \\
\text { Envelope spatial frequency }(0.05-0.4 \mathrm{cpd}) \\
\text { Figure: } 3\end{array}$ & $\begin{array}{l}\text { Increasing envelope spatial } \\
\text { frequency (increasing upper } \\
\text { sideband frequency) makes reversal } \\
\text { stronger and/or occurs at lower } \\
\text { contrasts. }\end{array}$ & yes \\
\hline $2.2 \mathrm{~A}$ & $\begin{array}{l}\text { The effect of stimulus location } \\
\text { Conditions: AM and beat; carrier, } 1 \mathrm{cpd} ; 10 \times \text { threshold; } 6^{\circ} \text { central } \\
\quad \text { disk; envelope, } 2 \times, 3 \times \text { threshold } \\
\text { Variables: Temporal drift rate }(0.4-16 \mathrm{~Hz}) \\
\text { Duration }(0.03125-0.5 \mathrm{sec}) \\
\text { Central and } 10^{\circ} \text { peripheral } \\
\text { Figures: } 4 \mathrm{~A}-4 \mathrm{~F}\end{array}$ & $\begin{array}{l}\text { Moving stimulus into periphery } \\
\text { makes reversal stronger and/or } \\
\text { occurs at lower contrasts. }\end{array}$ & yes \\
\hline $2.2 \mathrm{~B}$ & $\begin{array}{l}\text { The effect of stimulus spatial configuration } \\
\text { Conditions: AM; carrier, } 1 \mathrm{cpd} ; 2-\text { and } 16-\mathrm{Hz} \text { drift; envelope, } \\
\quad 100 \% \text { modulated } \\
\text { Variables: Carrier contrast, } 10 \times-200 \times \text { threshold } \\
\text { Duration }(0.03125-0.5 \mathrm{sec}) \\
20^{\circ} ; 4^{\circ} ; 20^{\circ} / 8^{\circ} \text { configuration } \\
\text { Envelope spatial frequency }(0.05-0.4 \mathrm{cpd}) \\
\text { Figures: } 5 \mathrm{~A}-5 \mathrm{C}\end{array}$ & $\begin{array}{l}\text { Moving stimulus into periphery } \\
\text { makes reversal stronger and/or } \\
\text { occurs at lower contrasts. }\end{array}$ & yes \\
\hline 2.3 & $\begin{array}{l}\text { The effect of envelope modulation depth (side-band phase) } \\
\text { Conditions: AM and QFM; carrier, } 1 \mathrm{cpd} ; 2 \text { - and } 16-\mathrm{Hz} \text { drift; duration, } \\
0.5 \mathrm{sec} \text {; envelope, } 100 \% \text { modulated } \\
\text { Variables: Carrier contrast, } 10 \times-200 \times \text { threshold } \\
20^{\circ} ; 4^{\circ} ; 20^{\circ} / 8^{\circ} \text { configuration } \\
\text { Envelope spatial frequency }(0.1 \text { and } 0.2 \mathrm{cpd}) \\
\text { Figures: } 6 \mathrm{~A}-6 \mathrm{E}\end{array}$ & $\begin{array}{l}\text { Changing from AM to QFM will } \\
\text { affect only forward motion, not } \\
\text { reversal. May have stronger } \\
\text { reversal in QFM due to forward/ } \\
\text { reversed motion balance in stimulus } \\
\text { changing. }\end{array}$ & yes \\
\hline 2.4 & $\begin{array}{l}\text { The effect of carrier color } \\
\text { Conditions: AM and QFM; carrier, } 1 \mathrm{cpd} \text {; duration, } 0.5 \mathrm{sec} \text {; } \\
\text { envelope, } 0.1 \mathrm{cpd} 100 \%(50 \%) \text { modulated } \\
\text { Variables: Carrier contrast, } 10 \times-200 \times \text { threshold } \\
\text { Temporal drift rate }(0.4-16 \mathrm{~Hz}) \\
\text { Duration }(0.03125-0.5 \mathrm{sec}) \\
20^{\circ} ; 4^{\circ} ; 20^{\circ} / 8^{\circ} \text { configuration } \\
\text { Figures: } 7 \mathrm{~A}-7 \mathrm{D}\end{array}$ & $\begin{array}{l}\text { Changing to a chromatic carrier } \\
\text { should reduce temporal frequency } \\
\text { at which reversal occurs; may see a } \\
\text { stronger reversal. }\end{array}$ & no \\
\hline 2.5 & $\begin{array}{l}\text { The effect of stimulus duration } \\
\text { Conditions: AM; carrier, } 1 \mathrm{cpd} ; 2-\text { and } 16-\mathrm{Hz} \text { drift; envelope, } \\
0.1 \mathrm{cpd} 100 \% \text { modulated } \\
\text { Variables: Carrier contrast, } 10 \times-200 \times \text { threshold } \\
\text { Duration }(0.03125-0.5 \mathrm{sec}) \\
20^{\circ} ; 4^{\circ} ; 20^{\circ} / 8^{\circ} \text { configuration } \\
\text { Figures: } 8 \mathrm{~A} \text { and } 8 \mathrm{~B}\end{array}$ & $\begin{array}{l}\text { Reversal should persist at short } \\
\text { durations, forward (envelope) mo- } \\
\text { tion will be diminished. }\end{array}$ & no \\
\hline
\end{tabular}

the greater the reduction in upper sideband visibility, the stronger the reversal that would be predicted from the window of visibility approach. The most likely effect of this, in terms of the data, is a performance level closer to $0 \%$ correct (reversed motion) in patterns with a higher spatial frequency envelope for a given temporal frequency and scaled contrast.

The data presented in Figure 3 plot the performance in the direction discrimination task against the contrast of the carrier of the AM stimulus, expressed in multiples of the threshold required to detect the carrier alone. Each data point plots the mean of 100 observations, and the error bars are $\pm 1 S E M$. As was discussed above, the envelope modulation is set at $100 \%$ to avoid any limitation attributable to modulation depth on performance. Four spatial frequency combinations are shown for observer R.D., two for C.L.W. The carrier is always $1 \mathrm{cpd}$; envelope spatial frequencies increase from 0.05 through 0.1 and 0.2 to $0.4 \mathrm{cpd}$. The effect of this increase is to move the sidebands further apart, either side of the carrier, in 
Observer D.M.

Envelope Twice Detection Threshold

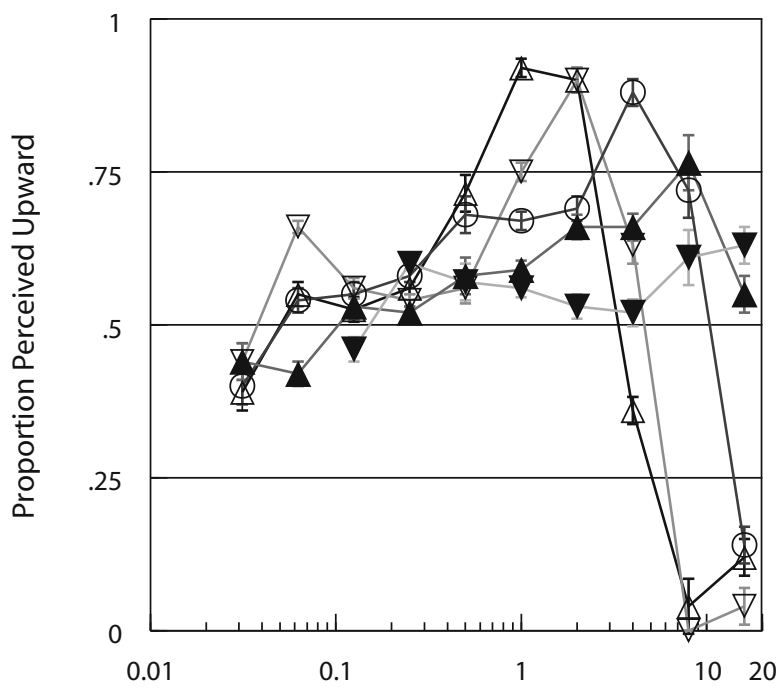

Temporal Frequency $(\mathrm{Hz})$
Observer D.M.

Envelope Thrice Detection Threshold

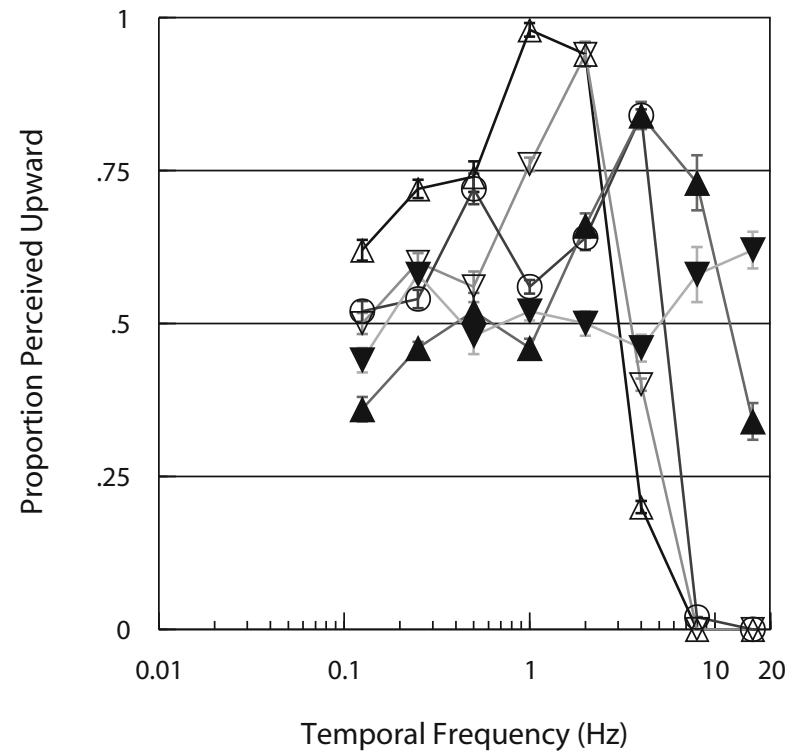

Observer S.J.C.

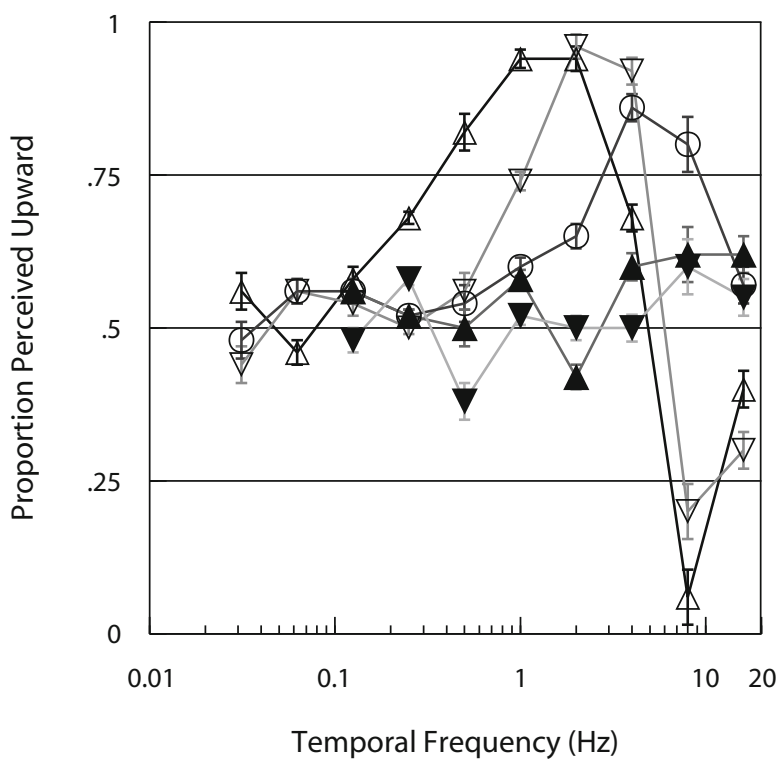

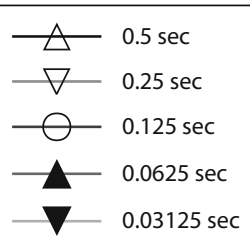

Figure 2. Experiment 1: Baseline data showing both forward and reversed motion as a function of temporal drift frequency and duration for an amplitude-modulated grating. Performance in the direction discrimination task (as a proportion correct) is plotted against the temporal drift frequency of the envelope. Each duration (half-width of the raised-cosine temporal envelope) is plotted as a separate data series as indicated in the key. Data for 2 observers are shown: D.M. at envelope modulation depths of twice and thrice discrimination threshold, S.J.C. at twice discrimination threshold. The carrier is set at $1.0 \mathrm{log}$ units (10 times) carrier detection threshold. In every condition presented in this article, all detection thresholds were measured at the appropriate stimulus duration. Error bars are $\pm S E M$, and there are at least 100 observations per data point throughout all figures.

frequency space. Two envelope temporal frequencies are plotted, 2 and $16 \mathrm{~Hz}$, which should give rise to forward and reversed motion, respectively (see Figure 2).

The envelope spatial frequency has little effect upon the forward motion perceived in the low temporal frequency condition (filled symbols), where both observers are near perfect performance at all carrier contrasts. It should be noted that lower carrier contrasts do not reduce performance significantly down to a carrier contrast $0.5 \log$ units above threshold (Cropper, 1994; Cropper \& Derrington, 1994). In the high temporal frequency condition (open symbols), however, the spatial frequency of the 
Observer C.L.W.

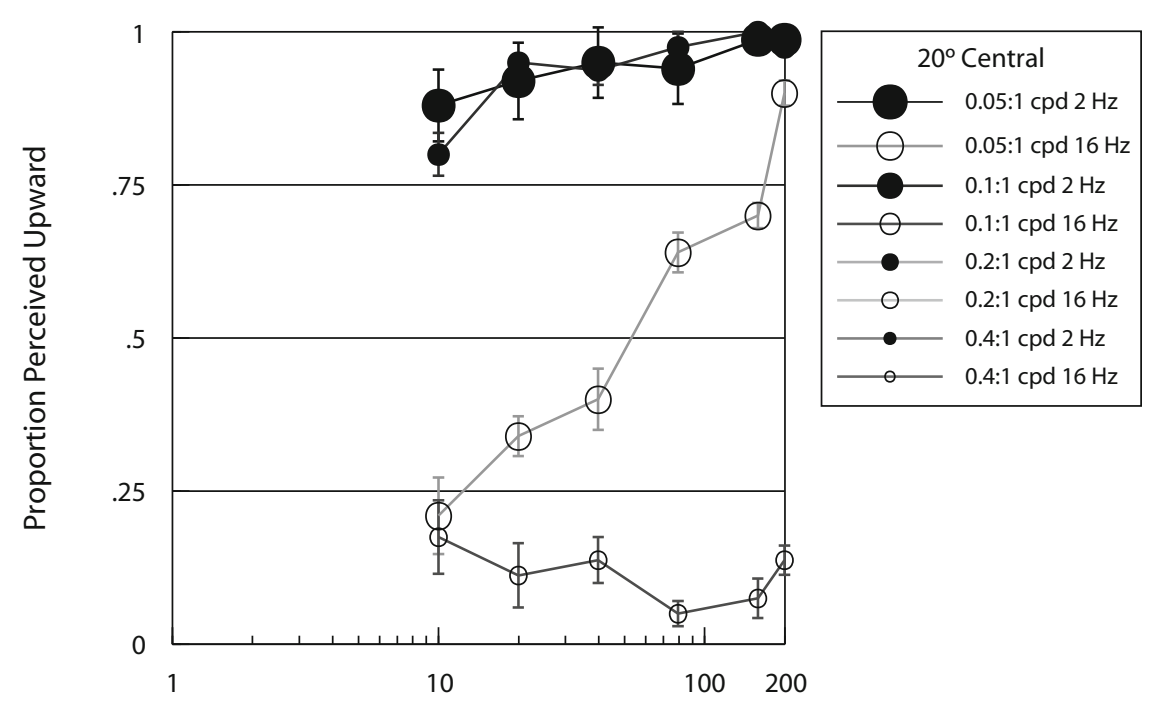

Carrier Contrast (Multiples Above Threshold)

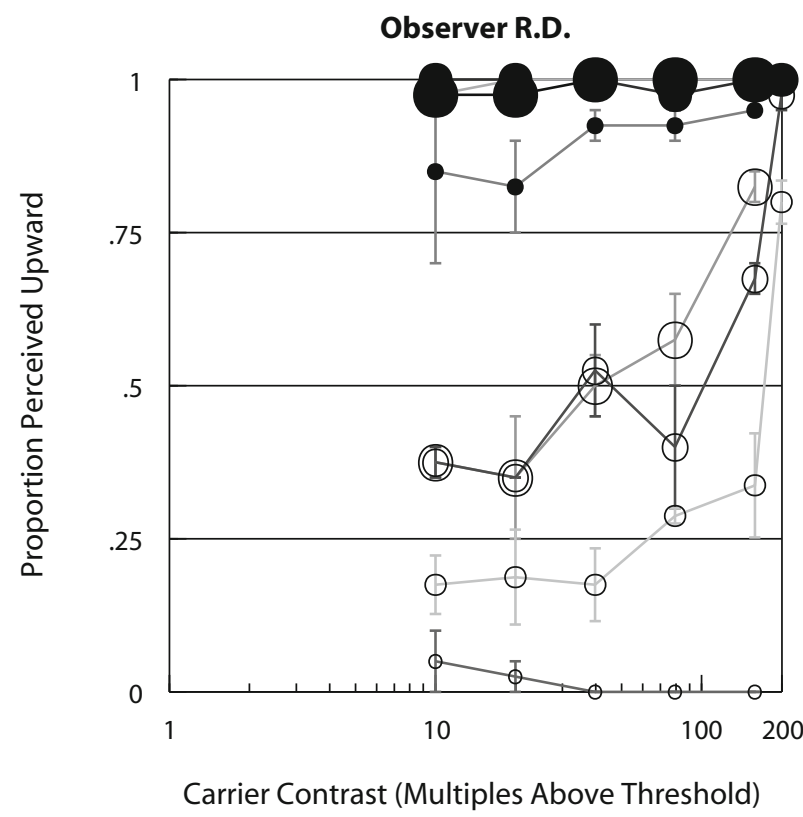

Figure 3. Experiment 2.1: Effect of spatial frequency (denoted as envelope:carrier) on direction discrimination. The proportion correct in the direction discrimination task is plotted against the carrier contrast, expressed as multiples of carrier detection threshold for each observer and duration. Data for two drift rates are shown: 2 and $16 \mathrm{~Hz}$ at several different spatial frequency combinations. Two observers are shown: C.L.W. and R.D. for a $20^{\circ}$ radius amplitude-modulated stimulus with a $100 \%$-modulated envelope.

envelope has a dramatic effect upon whether the envelope is seen to move in the direction opposite to its true motion or whether there is no consistent direction reported. As the spatial frequency of the envelope increases (indicated by the reduction in size of the symbols), so the proportion of perceived reversals increases at all contrasts. Related to this, as the carrier contrast increases, more forward motion is seen in the lower spatial frequency en- velope conditions. There is very little reversal, in fact, for the lower spatial frequency conditions of 0.1:1 and $0.05: 1 \mathrm{cpd}$, and under higher carrier contrast conditions, there is a recovery of veridical judgments, indicating that the observer sees forward motion for the lower spatial frequency envelopes. Observer R.D. shows more of this effect than does C.L.W. at an envelope spatial frequency of $0.2 \mathrm{cpd}$. This effect of spatial frequency upon a per- 
ceived reversal agrees, in general, with previous data (Wang et al., 1997).

The decrease in perceived reversal with increasing carrier contrast raises an important issue with regard to the window of visibility approach, although it is also an issue that must be addressed by any model claiming to predict performance. The issue is the long-standing one of the relationship between stimulus contrast, perceived direction and speed of motion, and the task required of the observer (Cropper, 1994; Johnston, Benton, \& Morgan, 1999; Stone \& Thompson, 1992; Thompson, 1982, 1984). As one might expect, the result of increasing the temporal frequency of the upper sideband and correspondingly decreasing its visibility is not an all-or-nothing effect. That is, by raising the upper sideband's contrast alongside the carrier contrast (keeping the ratio of 1:2:1), the sideband again becomes visible, and veridical direction perception is at least partially restored (Figure 3 ). What is also indicated by the data is that the perceived direction of the composite pattern is not dependent on the relative suprathreshold contrast of the two sidebands, since the lower sideband contrast is concurrently raised by the same amount as the upper sideband, keeping the numerical contrast ratio at 1:2:1. One would expect the reversal to persist if the suprathreshold contrast dictated the relative direction strength of the two. It seems, therefore, that the important factor-in particular, from a window of visibility approach - is that both sideband contrasts are suprathreshold for the envelope motion to be seen veridically. This observation is also consistent with previous data concerning the discrimination of direction in simple grating stimuli (Levinson \& Sekuler, 1975a, 1975b; Watson, Thompson, Murphy, \& Nachmias, 1980). On the other hand, a graded change in direction as a function of the higher contrasts for the 0.05- and 0.1-cpd envelopes shown in the data indicates that there is a restricted range over which the relative contrast of the two sidebands may matter in some component-frequency combinations. In summary, the conclusion one is inclined to reach from this manipulation of spatial frequency is that the reversal is most intuitively explained by the reduction of the visibility of the higher spatial frequency sideband as its temporal frequency increases.

Experiment 2.2: The effect of stimulus configuration and retinal location. Recent work has shown that as one moves the retinal location of a given stimulus into the periphery, the perception of both the structure and the motion of a second-order pattern is degraded in some way (Derrington \& Cox, 1998; Jamar et al., 1984; Pantle, 1992; Smith et al., 1994; Smith \& Ledgeway, 1998; Solomon \& Sperling, 1995). The quantitative effect of the move to the periphery upon the perception of the contrast-modulated envelope motion is equivocal and seems to depend heavily on stimulus parameters, such as carrier contrast, modulation depth, configuration, and temporal frequency. What is clear, however, is that the perception of the motion of a contrast envelope is affected by moving it away from central vision, a characteristic that it has in common with many other visual tasks.

As the spatial frequency of the envelope and the carrier approach each other in value (sidebands move farther apart in spatial frequency), it becomes much harder to discriminate a contrast-modulated carrier from its carrier presented alone (Cropper, 1998; Jamar et al., 1982). As the component spatial frequencies move apart, the likelihood of the spatial contrast sensitivity function affecting their visibility differentially is increased. This observation has been used to explain the reversal of motion of a contrast envelope presented in the periphery, the motion of which was seen veridically in central vision (Wang et al., 1997).

The basis for this suggestion is that there is a relatively greater reduction in contrast sensitivity in the periphery than in the central visual field as temporal frequency increases. This may be because the spatial summation process that enhances peripheral contrast sensitivity at the cost of spatial resolution (Green, 1970; Thibos, Cheney, \& Walsh, 1987; Virsu, Rovamo, Laurinen, \& Näsänen, 1982; Wang et al., 1997) is compromised by the increase in temporal frequency of the stimulus. Because central vision does not rely on summation in the same way as peripheral vision, the effect of increasing temporal frequency is not as severe.

Experiment 2.1 (above) presented data for stimuli presented centrally, which, given some reports (Wang et al., 1997), may be predicted to be perceived to move backward when located in the near periphery. In terms of the perspective offered by the window of visibility model, at lower temporal frequencies, the higher spatial frequency sideband will become less visible in the periphery than in central vision, the consequence of which is that the reversal will be seen at lower temporal frequencies in the periphery. These observations clearly indicate that stimulus size and extent are critical factors in the result, since the larger the stimulus, the further it extends into the periphery. These differences in stimulus size may well be the root of some of the disagreements in the literature to date. In Experiment 2.2, we examined whether there would be more motion reversal of the envelope in the periphery, as compared with central vision, as the temporal frequency of the envelope increased. Experiment 2.2 (supplemental; hereafter Experiment 2.2s) presents data comparing the initial conditions of a $6^{\circ}$ disk presented centrally and located $10^{\circ}$ to the left of fixation. For brevity, these data are presented as a supplement (see the online version of this article to download). The main experiment then was performed to examine the effect of comparing a $20^{\circ}$ disk with an annulus with an outer diameter of $20^{\circ}$, an inner diameter of $8^{\circ}$, and a small central disk of $4^{\circ}$. Thus, the two experiments $(2.2 \mathrm{~s}$ and 2.2$)$ covered most of the configurations in the current literature (Derrington \& Cox, 1998; Jamar et al., 1984; Pantle, 1992; Smith et al., 1994; Smith \& Ledgeway, 1998; Solomon \& Sperling, 1995). In Experiment 2.2, the contrast of the carrier was scaled to the sensitivity measured for a particular configuration. However, at a carrier contrast of $1.0 \mathrm{log}$ unit above threshold, slight deviations from this exact suprathreshold level, as may have been the case in Experiment 2.2s, in which contrast was scaled only to duration, are unlikely to make a measurable difference to performance (Cropper, 1998).

The following data show the effects of changing the stimulus configuration from a large $20^{\circ}$ disk, to an an- 
nulus $\left(20^{\circ}\right.$ outer, $8^{\circ}$ inner diameter), and to a small $4^{\circ}$ central disk. The purpose was to examine the differential response centrally and peripherally to the same stimulus. Each condition used contrast scaled to sensitivity for that particular stimulus. Contrast was used as the continuous independent variable, rather than temporal frequency (Experiment 2.2s); only two temporal frequencies, 2 and $16 \mathrm{~Hz}$, were presented, as in Experiment 2.1.

In order that the reversal would be minimized from the point of view of the window of visibility explanation, the principal spatial frequency combination used was a 0.1 -cpd envelope modulating a 1-cpd carrier. Thus, the sinusoidal components of the AM grating were close together in spatial frequency ( 0.9 and $1.1 \mathrm{cpd})$, reducing the differential visibility due to temporal frequency and the contrast sensitivity function. Any effect of the retinal location of the stimulus upon the reversal should then be given maximum opportunity to become apparent.

Figure 4A shows data for 4 observers performing the direction discrimination task for a 0.1 -cpd envelope modulating a 1 -cpd carrier (components, $0.9,1.0$, and $1.1 \mathrm{cpd}$ ). The three stimulus configurations $\left(20^{\circ}, 20^{\circ} / 8^{\circ}\right.$, and $\left.4^{\circ}\right)$ are indicated by the different symbol shapes; filled symbols indicate an envelope drift frequency of $2 \mathrm{~Hz}$, open symbols indicate a drift rate of $16 \mathrm{~Hz}$. Observers R.D. and L.K. performed all the conditions; S.J.C. and C.L.W. performed two conditions each. There was very little effect of the stimulus configuration on the perceived duration of motion at a temporal frequency of $2 \mathrm{~Hz}$ for all the observers: The stimuli were all seen as moving forward most of the time. Observers L.K. and S.J.C. showed a strong effect of stimulus configuration upon the perceived reversal: The strongest reversal was perceived in the annular condition; the least reversal was perceived in the small central condition. This general trend was similar to that exhibited by S.J.C. and D.M. in Experiment 2.2s; as the stimulus became more centrally localized, less reversal was perceived at high temporal drift frequencies. Observer R.D. showed a worse performance than did L.K. in the $20^{\circ}$ and $20^{\circ} / 8^{\circ}$ conditions, although the general trend was similar. Observer C.L.W. exhibited a performance similar to that of R.D. for the $20^{\circ}$ and $20^{\circ} / 8^{\circ}$ conditions; this is consistent with Experiment 2.2s and with a comparison of the performance of Observers C.L.W. and R.D.

Figure 4B presents data for Observer R.D. with spatial frequency combinations of $0.4: 1 \mathrm{cpd}$ (components, $0.6,1.0$, and $1.4 \mathrm{cpd}$ ), 0.2:1 cpd (components, 0.8, 1.0, and $1.2 \mathrm{cpd}$ ), and $0.05: 1 \mathrm{cpd}$ (components, $0.95,1.0$, and $1.05 \mathrm{cpd}$ ). Forward motion in the $2-\mathrm{Hz}$ condition was principally unaffected by the configuration. The three spatial frequency combinations showed degrees of reversal similar to those in Experiment 2.1; more reversal was seen as the components moved further apart $(0.4: 1 \mathrm{cpd})$, and the reversal progressively decreased as the components moved nearer together (Wang et al., 1997). The relative effect of the stimulus configuration within each spatial frequency combination is not clear-cut across the different spatial frequency combinations, but there is a suggestion of less reversal perceived in the $4^{\circ}$ central condition than in either the $20^{\circ}$ or the $20^{\circ} / 8^{\circ}$ annulus configuration.
The effect of temporal frequency upon two spatial frequency configurations $(0.1: 1$ and $0.2: 1 \mathrm{cpd})$ in the near periphery $\left(20^{\circ} / 8^{\circ}\right.$ annulus $)$ condition is shown in Figure 4C for Observer R.D. The performance in the direction discrimination task is plotted against the temporal drift frequency of the envelope (note the use of a linear $x$-axis, as compared with earlier temporal frequency figures, in order that the data points would be evenly spread). Both spatial frequency combinations showed a reduction in (forward) performance as the temporal frequency increased, which resulted in a reversal in some cases. For a given temporal frequency, a greater reversal was seen for the 0.2:1-cpd combination. As contrast of the carrier (and the components) increased from $1.3 \mathrm{log}$ units above threshold to $2.3 \log$ units above threshold, the degree to which the stimulus was seen to reverse was reduced for each spatial frequency combination.

It would be an overstatement to say that the data from Experiments 2.2s and 2.2 are conclusive evidence that the reversal of motion perceived in the high temporal frequency stimuli was localized in the near periphery, but there is a strong suggestion that this was the case, particularly from Observers L.K. and S.J.C. However, unsurprisingly, the effect is not so clear-cut as one might like, as is evident from the data for Observers C.L.W. and R.D. Previous work has shown that a peripheral reversal of motion occurs for lower $(4-\mathrm{Hz})$ temporal frequencies when the envelope and carrier are less than a factor of six apart in spatial frequency (the correlate of moving the components apart in spatial frequency; Wang et al., 1997). The present data show that this extends to lower relative spatial frequencies when the temporal frequency of the components is increased.

The data do support the basic prediction of the window of visibility model, in that the reversal seems to be stronger in the periphery than in the central visual field. The predictions that the reversal would occur at a lower temporal frequency in the periphery than in the central visual field (Experiment 2.2s), because of the differential effect of temporal frequency on sensitivity between the two is, however, not supported. Thus, one can only say there is partial support for such an approach. It is worth noting that previous work did not show any more support for the window of visibility approach than is shown here (Wang et al., 1997), since temporal frequency was not used as an independent variable.

Experiment 2.3: The effect of envelope modulation depth: AM and QFM gratings. The most intuitive test of the simple predictions made by the window of visibility model and the component-based approach, with respect to the present issue, would be to independently manipulate the components and the composite stimulus. This is hard to do, since the components govern the resultant composite stimulus, but there is a simple manipulation that retains the component contrast, spatial frequency, and temporal frequency yet changes the carrier-to-envelope relationship and the overall appearance of the composite stimulus. A QFM grating (Equation 6 in online supplemental materials) has exactly the same components as an AM grating, but the sideband spatial phase is shifted relative to the car- 


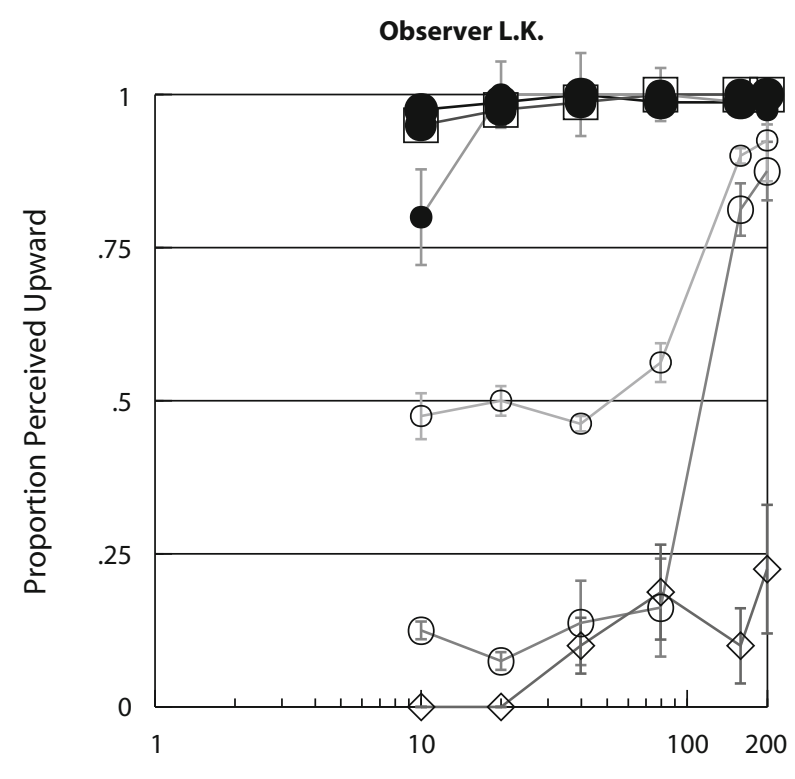

Carrier Contrast (Multiples Above Threshold)

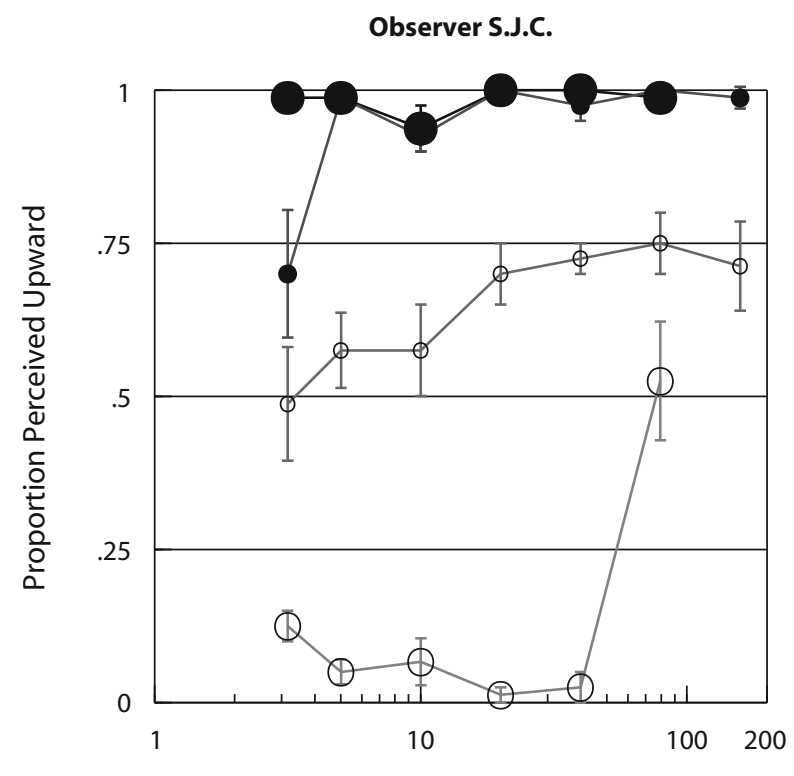

Carrier Contrast (Multiples Above Threshold)

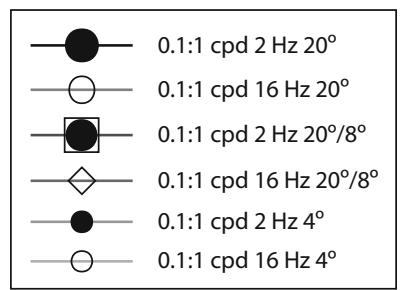

Observer R.D.

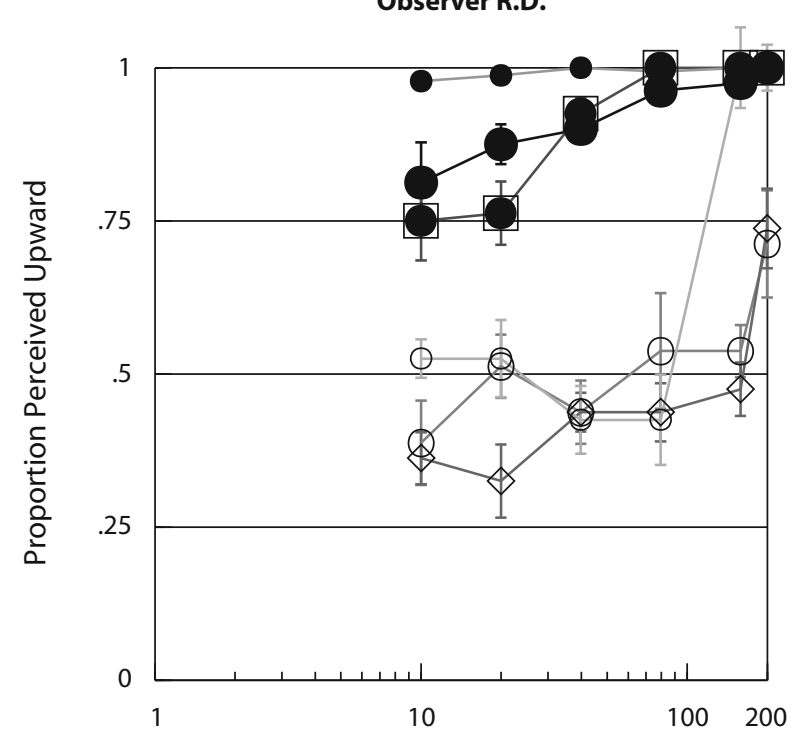

Carrier Contrast (Multiples Above Threshold)
Observer C.L.W.

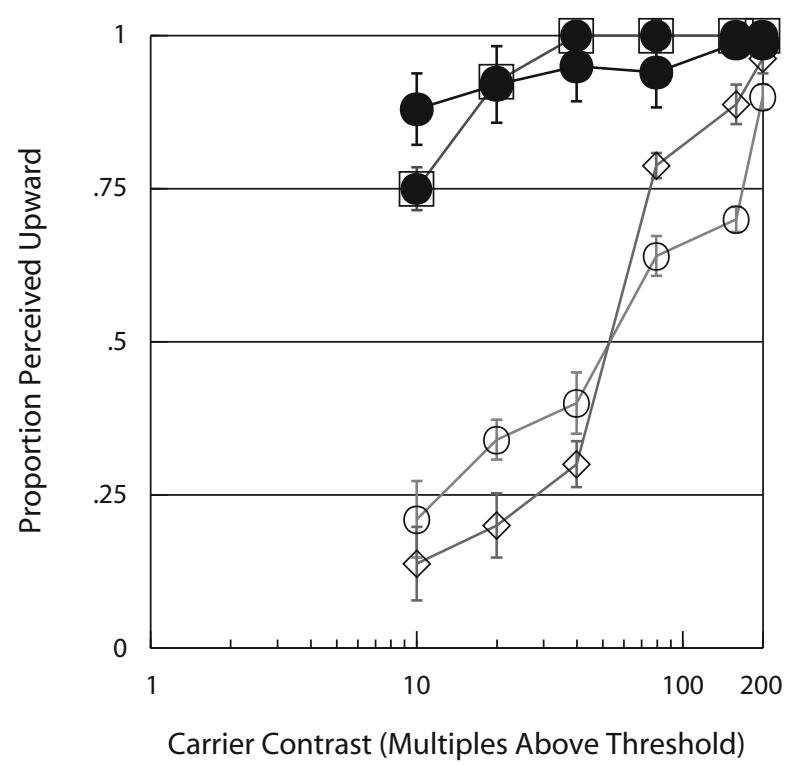

Figure 4A. Experiment 2.2: Effect of stimulus configuration on amplitude-modulated direction discrimination. The proportion correct in the direction discrimination task is plotted against the carrier contrast expressed as multiples of carrier detection threshold for each observer and condition. Stimuli are configured as a $20^{\circ}$ disk, a $4^{\circ}$ disk, or a $20^{\circ} / 8^{\circ}$ annulus, all centrally placed. Carrier contrast is $1.0 \mathrm{log}$ units above detection threshold, and envelope is $100 \%$ modulated and rifted at either 2 or $16 \mathrm{~Hz}$. Data from 4 observers are shown, each on a separate plot. 


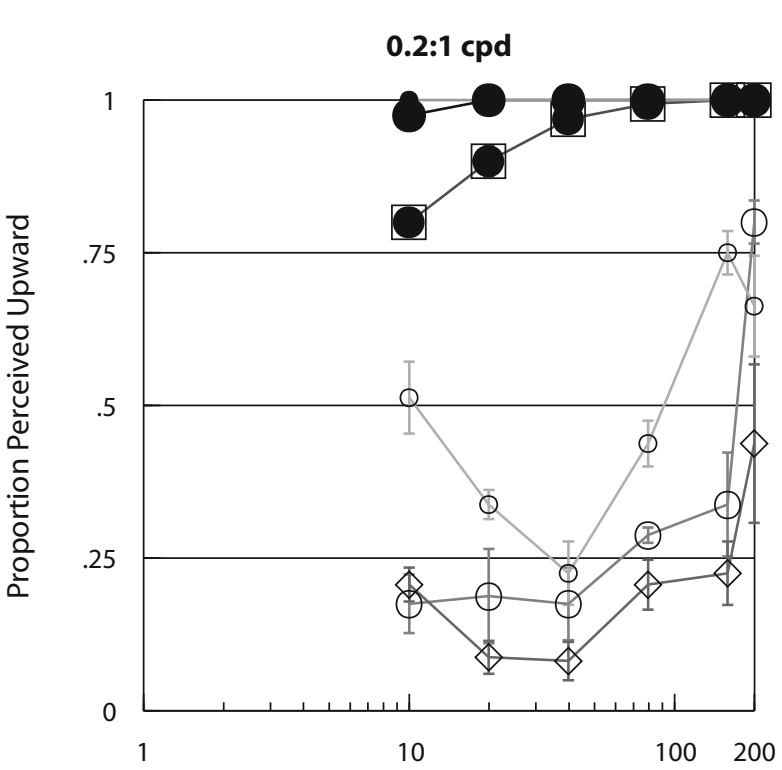

Carrier Contrast (Multiples Above Threshold)

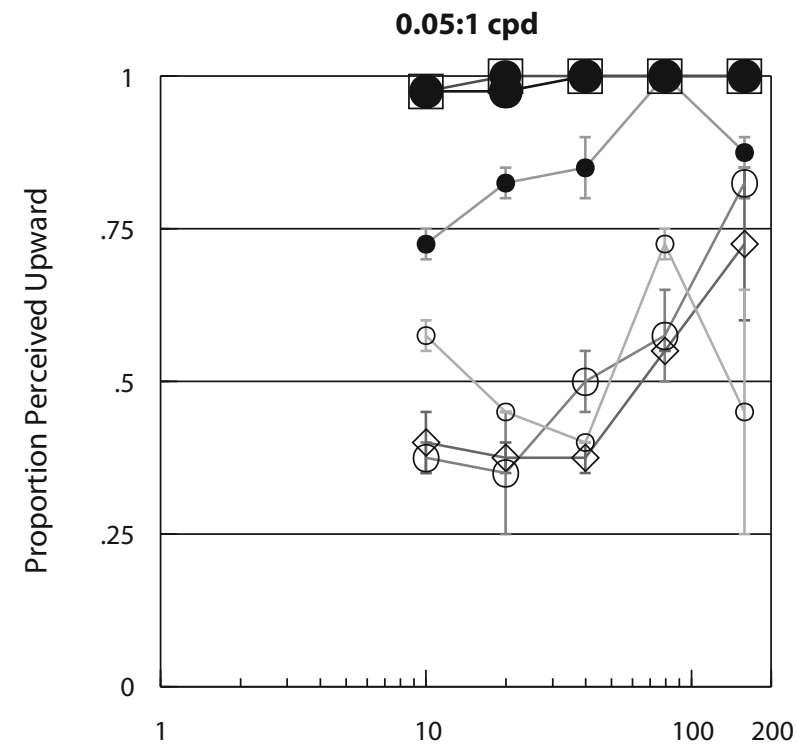

Carrier Contrast (Multiples Above Threshold)
$0.4: 1 \mathrm{cpd}$

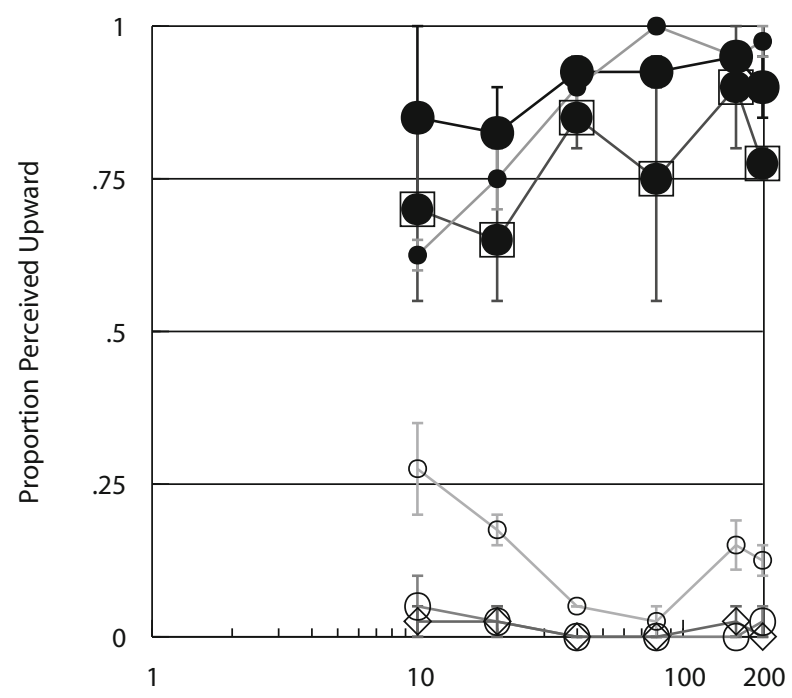

Carrier Contrast (Multiples Above Threshold)

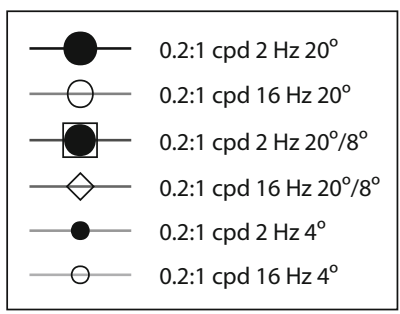

Figure 4B. Experiment 2.2: Conditions similar to those shown in Figure 4A, except that the envelope spatial frequency was changed from 0.1 cpd (Figure 5A) to 0.2, 0.4, and 0.05 cpd. Observer, R.D.

rier phase by a quarter of a cycle $(\pi / 2)$. The resultant pattern is, as is shown in Figure 1C, a carrier grating whose contrast is modulated to far less a degree than in an AM grating consisting of the same components but whose spatial frequency is modulated around the mean carrier frequency (Cropper, 1998; Henning et al., 1975).

The principal aim of Experiment 2.3 was to compare the discrimination of motion in otherwise equivalent AM and QFM patterns. A prediction of performance based on the components of the pattern would be similar for both gratings; performance based on the compound pattern would not necessarily be the same for AM and QFM. Thus, one might expect forward motion to be compromised in the QFM, as compared with the AM, pattern, with reversed motion left unaffected. A full data set was presented for Observer R.D. (0.2:1- and 0.1:1-cpd spatial frequencies in each of the three stimulus configurations for AM and QFM patterns), with confirmatory data from 2 other observers, C.L.W. and L.K.

The ability to discriminate the direction of the grating at a temporal frequency of $2 \mathrm{~Hz}$ (closed symbols) was severely compromised by changing the pattern from an 


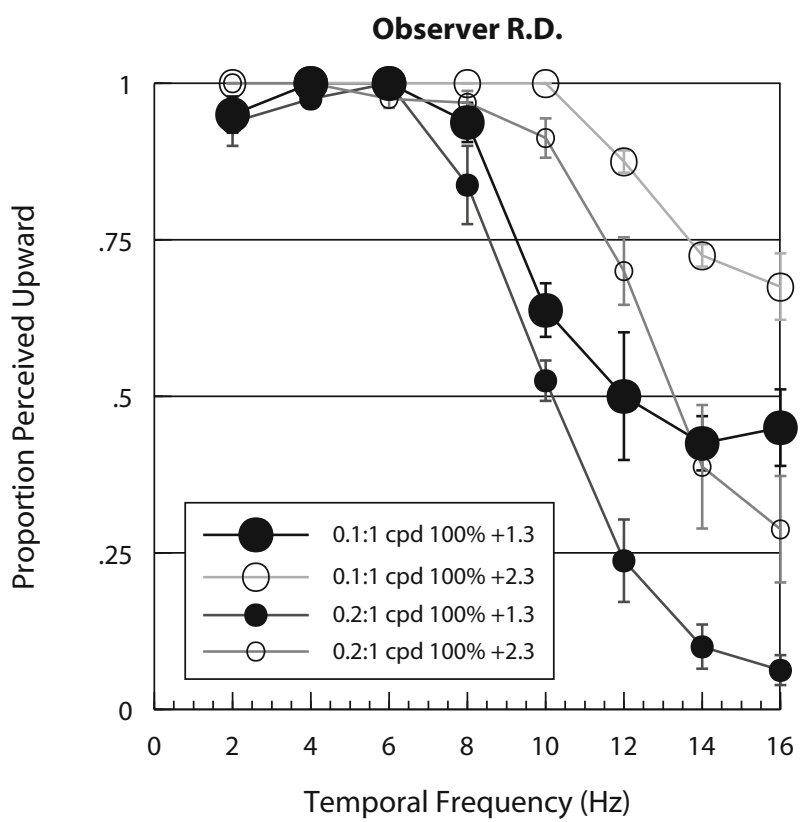

Figure 4C. Experiment 2.2: Examination of the effect of temporal frequency on direction discrimination at two envelope $(100 \%$ modulated) spatial frequencies $(0.1$ and $0.2 \mathrm{cpd})$ and two carrier $(1 \mathrm{cpd})$ contrasts $(1.3 \mathrm{log}$ units above threshold and $2.3 \mathrm{log}$ units above threshold) for Observer R.D.

AM grating to a QFM grating. There was either a reduction in performance toward chance levels for the $20^{\circ}$ (see Figure $5 \mathrm{~A}$ ) and $20^{\circ} / 8^{\circ}$ annulus (see Figure $5 \mathrm{~B}$ ) conditions or a reversal in perceived direction at this low temporal frequency in the $4^{\circ}$ condition (see Figure $5 \mathrm{C}$ ). The reversal was most likely due to the local motion of the carrier as the envelope drifted over it. When the stimulus was small, as in the $4^{\circ}$ case, this became a powerful cue. This is supported by the reduction in the reversal and a return to chance performance as the spatial frequency of the envelope increased; this caused the effective useful area of the stimulus to be increased as more envelope cycles became visible.

The high temporal frequency conditions (open symbols) showed very similar performance for the AM and the QFM patterns: Most conditions showed either a reversal in motion or chance performance. The important point is that the change from AM to QFM had little effect on performance in the $16-\mathrm{Hz}$ direction discrimination task but a very strong effect on the low temporal frequency $(2-\mathrm{Hz})$ condition.

In the lower graph of Figure 5D, the effect of decreasing the envelope depth of modulation is shown for observer L.K. The gray shaded symbols are the equivalent to the black symbols, except that the envelope modulation depth was $50 \%$. Performance was principally the same, except that the reduction in modulation depth had a much greater effect upon the percept of forward motion than upon that of reversed motion. If one adopts the approach that the critical factor in the reversal is the differential visibility of the lower sideband, as compared with the upper sideband, the reduction in envelope modulation depth would indeed be predicted to have a much greater effect on the perceived forward motion of the envelope, which was now half as deep. The relative visibility of the two sidebands remains the same, since their physical contrast remains the same as each other. This result supports the window of visibility

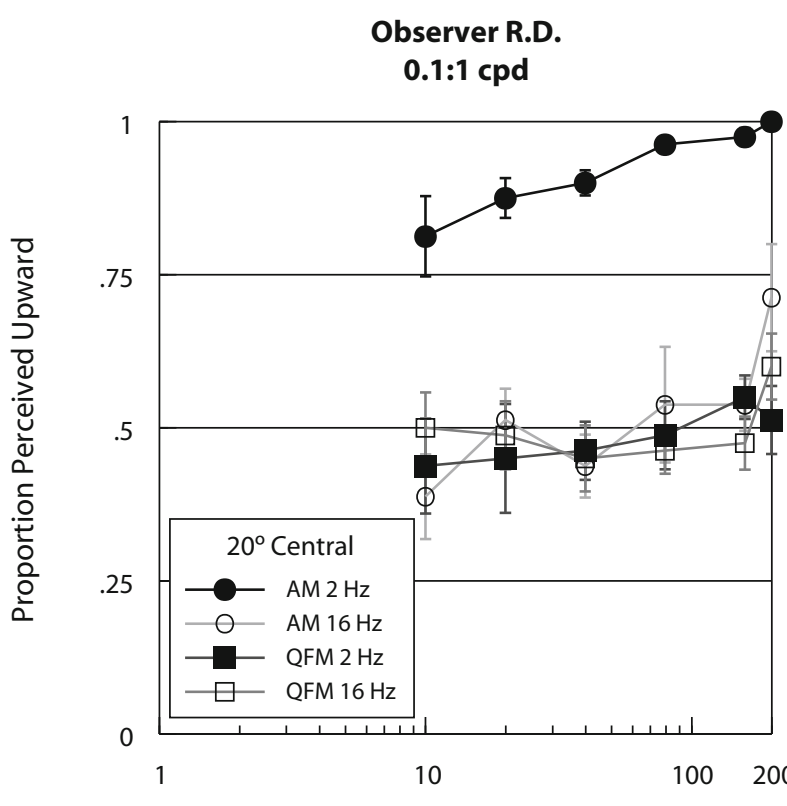

Carrier Contrast (Multiples Above Threshold)

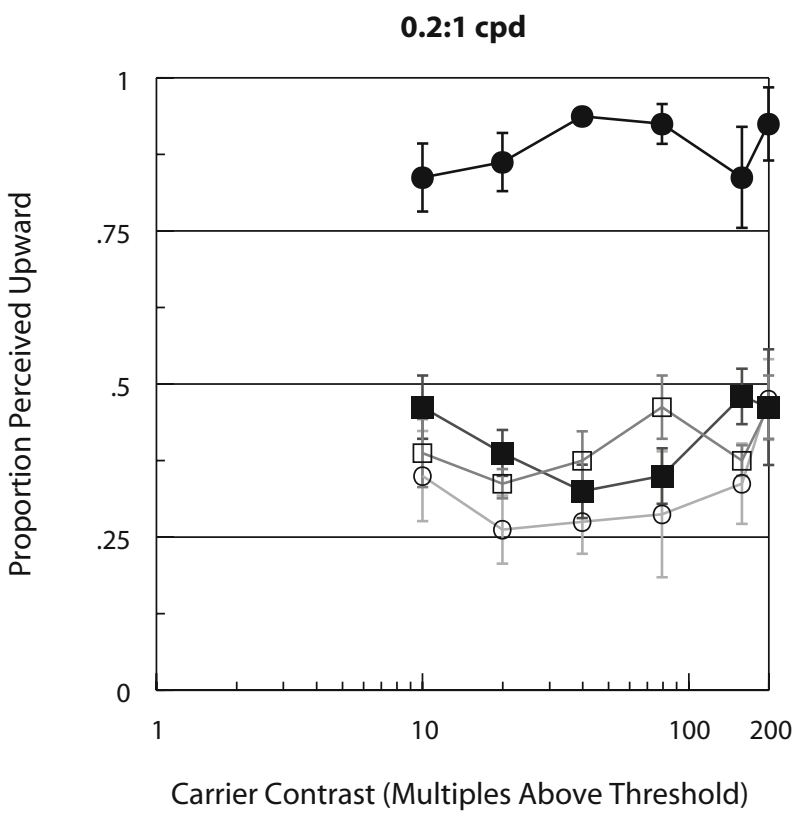

Figure 5A. Experiment 2.3: A comparison of direction discrimination performance in amplitude-modulated (AM) and quasifrequency-modulated (QFM) gratings. The proportion correct in the direction discrimination task is plotted against the carrier contrast expressed as multiples of carrier detection threshold for each observer and duration. The carrier was $1 \mathrm{cpd}$ and the envelope 0.2 cpd for both patterns, which were drifted at 2 and $16 \mathrm{~Hz}$. A $20^{\circ}$ disk configuration was used. Observer, R.D. See Experiment 2.3 for further details. 

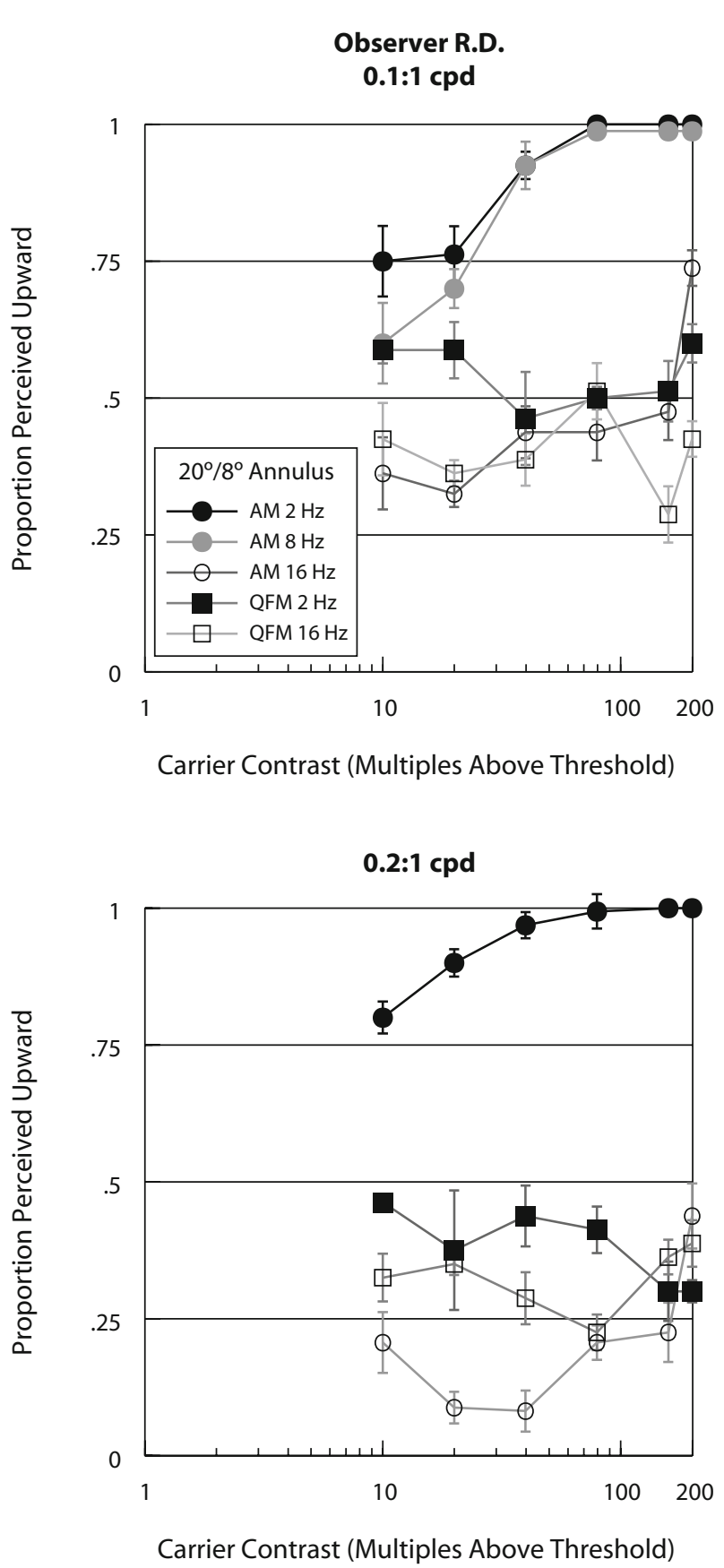

Figure 5B. Experiment 2.3: Details as in Figure 5A, except that the configuration was a $20^{\circ} / 8^{\circ}$ annulus.

as an explanation of the reversal but leaves the question of the relationship between the components and perception of forward motion open (Henning et al., 1975).

Experiment 2.4: The effect of carrier color. Previous work has shown that once a carrier is suitably scaled to detection threshold, whether it is a chromatic- or a luminance-modulated carrier has little effect upon the ability to detect or discriminate motion in a contrast envelope at moderate temporal frequencies (Cropper, 1998; Cropper \& Derrington, 1996). This result provides another po- tential test of the window of visibility explanation for the reversal observed in the luminance gratings thus far. Since the chromatic contrast sensitivity function falls off more quickly as a function of temporal frequency than does a luminance contrast sensitivity function (Mullen, 1985; Robson, 1966), the simple prediction would be to expect a stronger perceived reversal, which might well occur at a lower temporal frequency in a chromatic AM grating. A composite approach to the problem does not predict any difference between chromatic and luminance carriers.

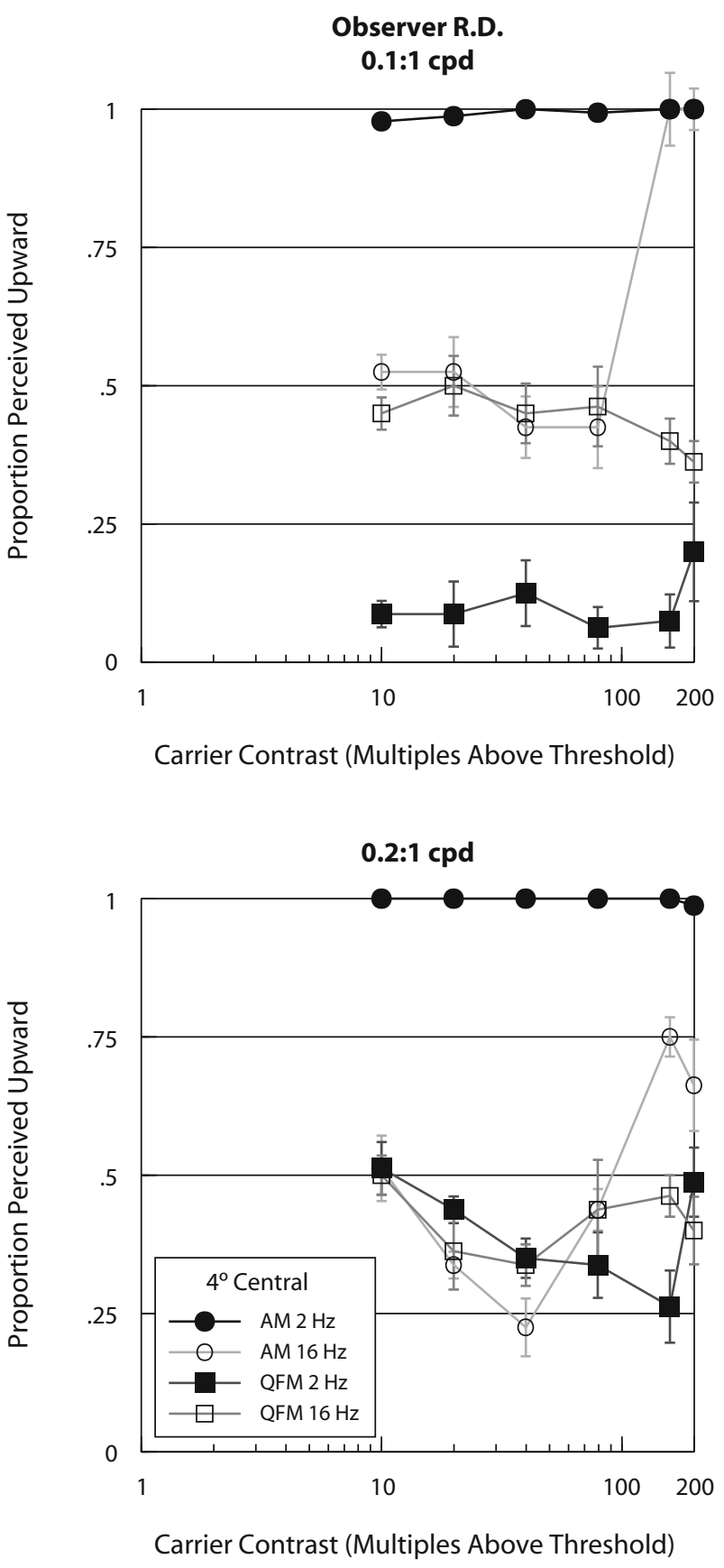

Figure 5C. Experiment 2.3: Details as in Figure 5A, except that the configuration was a $4^{\circ}$ disk. 

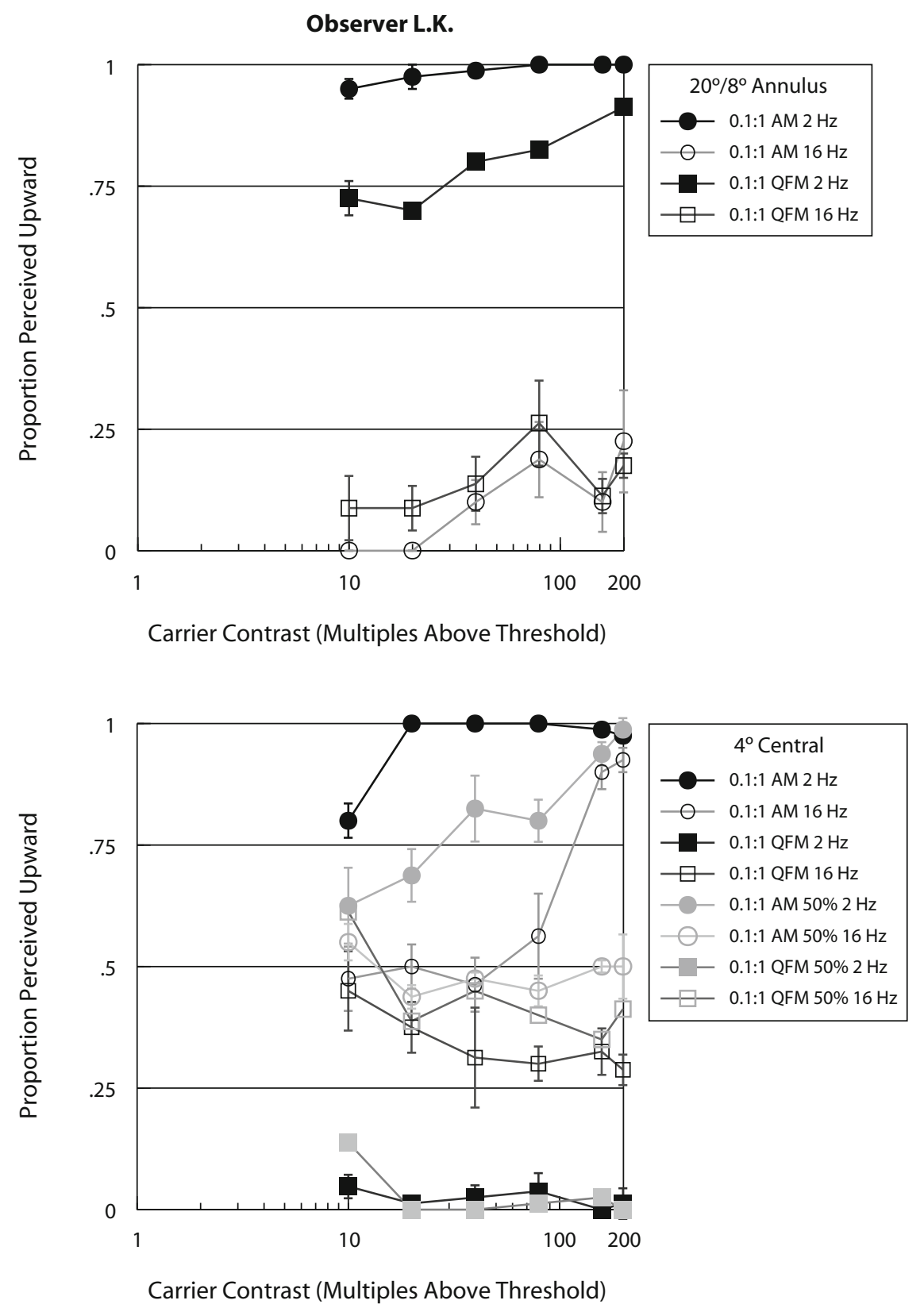

Figure 5D. Experiment 2.3: A comparison of AM and QFM across several critical conditions for Observer L.K. General details as in Figure 5A.

Figure 6A plots the performance in the direction discrimination task as function of envelope temporal frequency for two observers, S.J.C. and D.M. The carrier was an equiluminant $\mathrm{L}-\mathrm{M}$ grating $(1 \mathrm{cpd})$ with a contrast set at $1.0 \log$ units above detection threshold. The depth of amplitude modulation $(0.2 \mathrm{cpd})$ was set at twice discrimination threshold (approximately 50\%; Cropper, 1998). The most noticeable aspect of the data is that there seems to have been little or no reversal at the high temporal frequencies; performance dropped only to chance. Performance was generally poor at short durations and improved slightly as duration increased. Adequate forward motion - for instance, to es- tablish a threshold - was seen only for the longest duration of $0.5 \mathrm{sec}$. This is consistent with previous data (Cropper \& Derrington, 1996) and illustrates the only significant difference between chromatic and luminance carriers. From the point of view of the present emphasis upon reversal at high temporal frequencies, the lack of a reversal at any duration is a surprising result and contrary to the predictions made by the window of visibility model, since the differential visibility as temporal frequency increases should be exaggerated for the chromatic components.

Figures $6 \mathrm{~B}$ and $6 \mathrm{C}$ present data further examining this issue. Two temporal drift frequencies are presented for 
Observer D.M.

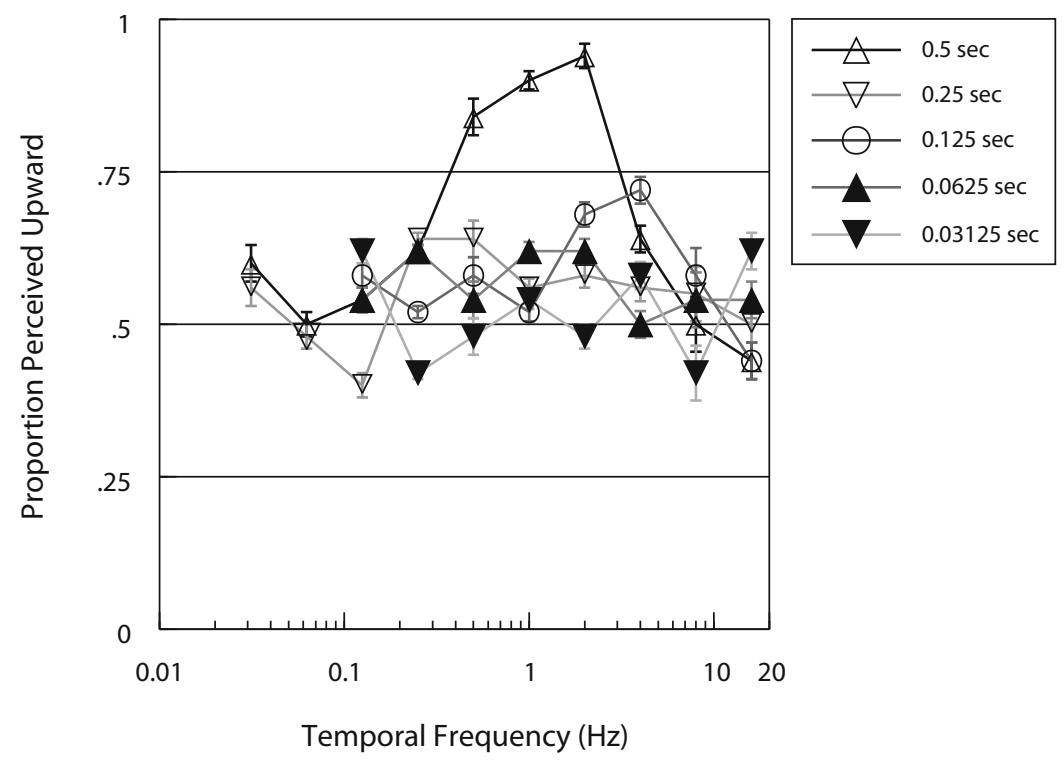

Observer S.J.C.

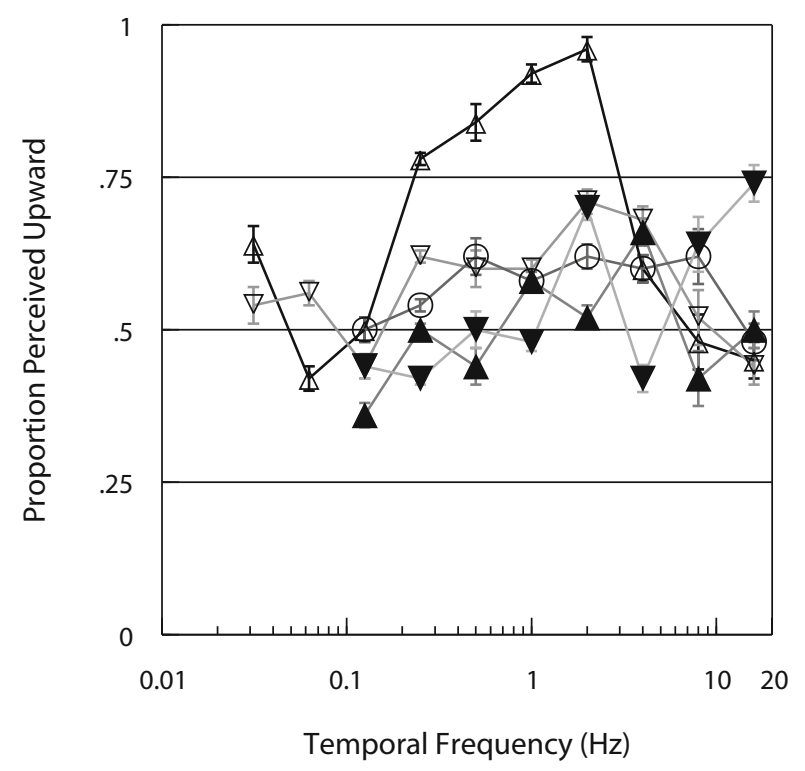

Figure 6A. Experiment 2.4: An examination of the effect of carrier chromaticity on envelope direction discrimination. Performance in the direction discrimination task (as a proportion correct) is plotted against the temporal drift frequency of the envelope. Each duration (half-width of the raised-cosine temporal envelope) is plotted as a separate data series as indicated in the key. Data for 2 observers are shown for a subjectively equiluminant $\mathrm{L}-\mathrm{M}$ carrier $(1 \mathrm{cpd})$ at a contrast of $1.0 \mathrm{log}$ units above detection threshold. Envelope ( $0.2 \mathrm{cpd})$ was modulated at twice modulation threshold. The data are comparable to those for the luminance carriers in Figure 2. Further details can be found in the text for Experiment 2.4.

the $100 \%$ modulated $\mathrm{AM}$ grating with either an $\mathrm{L}-\mathrm{M}$ or luminance carrier for 2 observers, L.K. and S.J.C. (for whom data for an L-M QFM grating is also shown). Performance is plotted against carrier contrast (in multiples above detection threshold). Shaded gray symbols indicate data for a luminance carrier; black symbols indicate data for an L-M carrier. The data for S.J.C. are plotted in Figure 6B, and those for Observer L.K. in Figure 6C. The two configurations are a full $20^{\circ}$ stimulus and a $4^{\circ}$ centrally placed stimulus. Again, the carriers are appropriately 


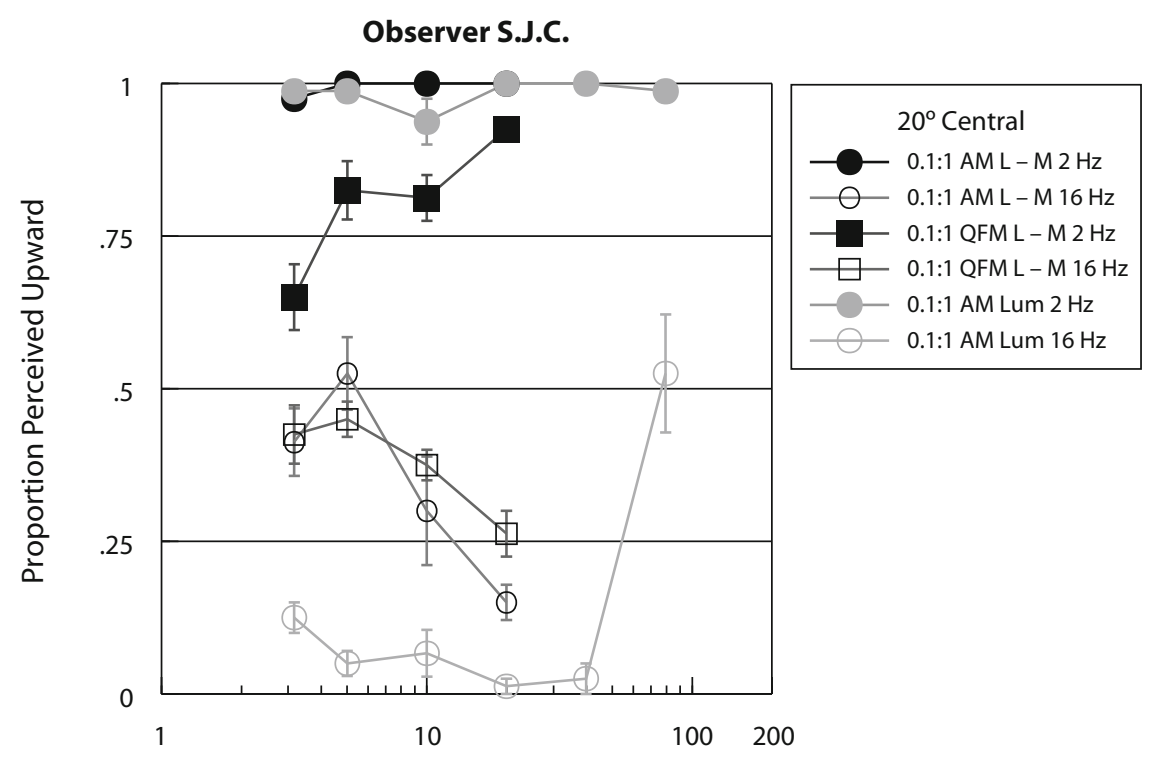

Carrier Contrast (Multiples Above Threshold)

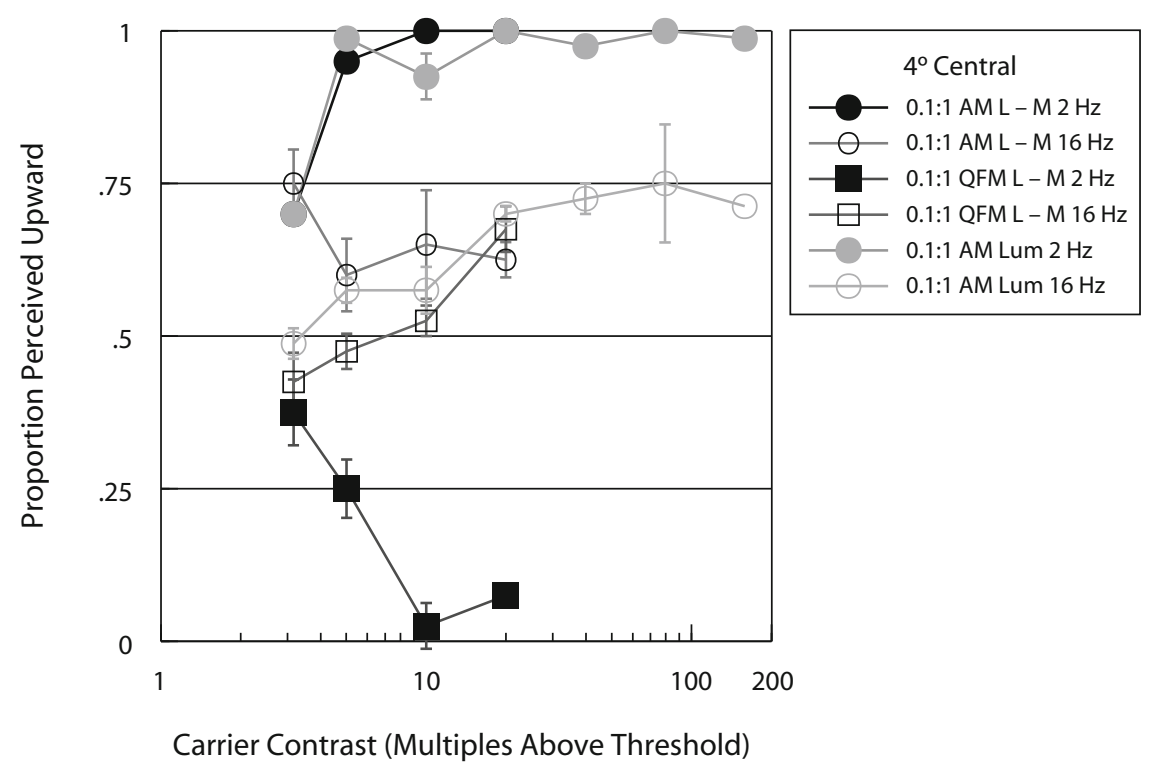

Figure 6B. Experiment 2.4: A comparison of stimulus color and configuration. The proportion correct in the direction discrimination task is plotted against the carrier contrast expressed as multiples of carrier detection threshold for each observer and duration. Both amplitude-modulated (AM) and quasi-frequency-modulated (QFM) gratings are shown, each drifted at 2 and $16 \mathrm{~Hz}$; luminance carriers are included for comparison. The top graph plots data for a $20^{\circ}$ disk; the lower graph plots data for a $4^{\circ}$ disk; both are centrally placed. The carrier is $1 \mathrm{cpd}$ and at a contrast of $1.0 \mathrm{log}$ units above threshold; the envelope is $0.2 \mathrm{cpd}$ and $100 \%$ modulated. Observer, S.J.C.

scaled to detection threshold for each configuration. Figure 6 C plots data for L.K. for a $20^{\circ} / 8^{\circ}$ annulus and for a $4^{\circ}$ central disk at an envelope modulation depth of $50 \%$.

In both the $20^{\circ}$ disk and $20^{\circ} / 8^{\circ}$ annulus conditions, there was less of a reversal of the envelope drifting at $16 \mathrm{~Hz}$ for a chromatic than for a luminance carrier at a given multiple of contrast detection threshold. At the same contrast, there was similar performance for the $2-\mathrm{Hz}$ condi- tion, where forward motion was seen to some degree in all the conditions. In the $4^{\circ}$ condition, there was very little reversal for either the $\mathrm{L}-\mathrm{M}$ or the luminance carrier, consistent with the configuration data in Experiment 2.2. The only perceived reversal was for the QFM grating, which is consistent with the data presented in Experiment 2.3. Figure 6D shows little difference in the performance for a $20^{\circ} / 8^{\circ}$ annulus, as compared with the $20^{\circ}$ central disk 


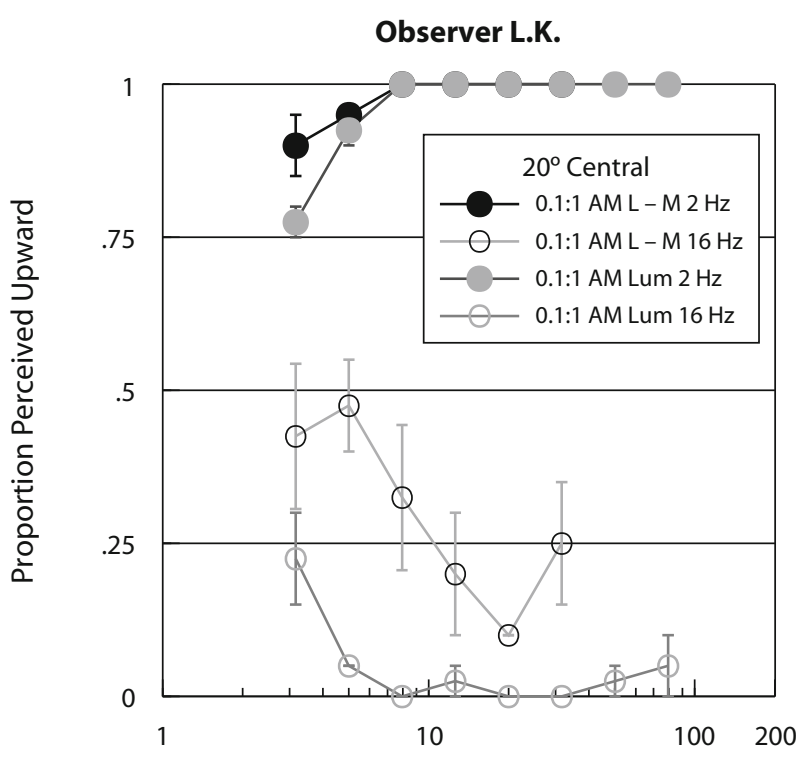

Carrier Contrast (Multiples Above Threshold)

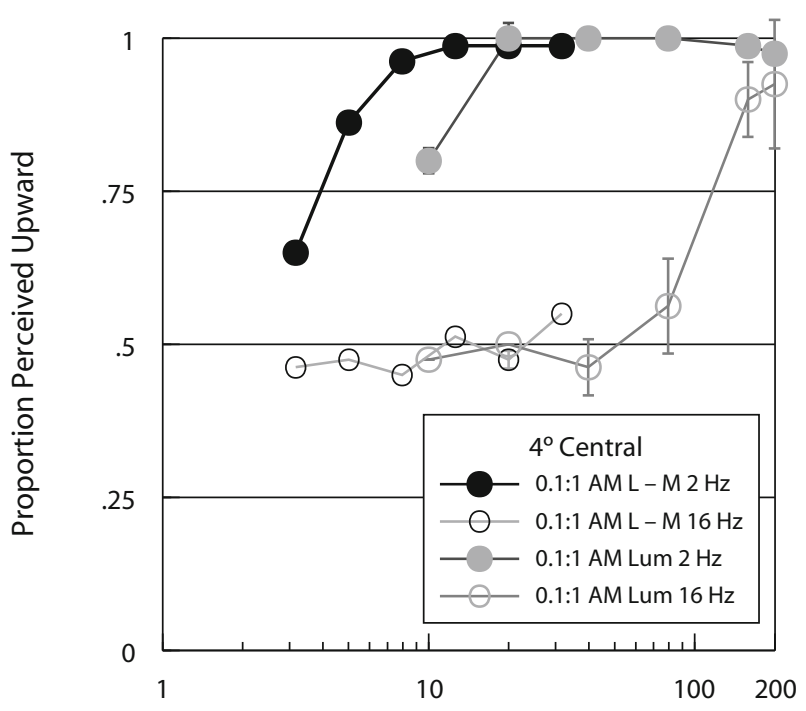

Carrier Contrast (Multiples Above Threshold)

Figure 6C. Experiment 2.4: Details as in Figure 6B, except that the stimuli are only AM stimuli and the observer is L.K.

(Observer L.K. only). At first blush, this is somewhat surprising, considering the specialization of the central visual field for chromatic processing. However, considering that the carrier contrast is scaled to detection threshold for each configuration, and that the majority of differences between the processing of the motion of luminance and chromatic patterns seem to be confined to first-order patterns only (Cropper, 1998; Cropper \& Derrington, 1994, 1996), the result is not so counter to expectations.

In terms of the predictions offered by the window of visibility model, the data with the chromatic carriers are contrary to the simple prediction that more reversal will be perceived in these patterns. This cannot simply be an issue of high temporal frequency chromatic components being less visible (Mullen, 1985), since no reversal can be seen at any temporal frequency in Figure 6A.

Experiment 2.5: The effect of stimulus duration. Finally, a fair proportion of the work concerned with the discrimination of motion of contrast-modulated patterns has shown that there seems to be some delay before the direction of motion of these patterns can be reliably discriminated. The outcome of this delay is that the direction of motion cannot be discriminated in second-order patterns at short durations (see Experiment 1) (Cropper \& Derrington, 1994, 1996; Derrington et al., 1993; Yo \& Wilson, 1992). This result has been explicitly incorporated into one of the more popular models of the perception of motion of second-order patterns (Wilson et al., 1992). If the reversal perceived at high temporal frequencies is solely due to the relative visibility of the sideband components in the pattern, the properties of the reversal should be consistent with other properties of the motion of first-order stimuli, one critical aspect of which is that the direction is discriminable at durations less than $0.01 \mathrm{sec}$ (Cropper \& Derrington, 1994, 1996). In Experiment 2.5, we examined this by reducing the duration of presentation of the stimulus while keeping the carrier contrast scaled to detection threshold at that duration. The window of visibility approach predicts that reversed motion will persist at short stimulus durations; existing data suggests that forward motion will disappear.

Figure 7A presents direction discrimination data for 2 observers, L.K. and C.L.W., for an AM grating $(0.1: 1 \mathrm{cpd}$, carrier contrast +1.0$)$ presented in a $20^{\circ} / 8^{\circ}$ annulus for different durations $(0.5,0.0625$, and $0.0312 \mathrm{sec})$. The annular arrangement was chosen so as to maximize the perceived reversal in order to best examine the effect of stimulus duration on the percept. The reduction in symbol size correlates to the reduction in stimulus duration; closed symbols indicate a drift rate of $2 \mathrm{~Hz}$, open symbols indicate a drift rate of $16 \mathrm{~Hz}$. As the duration of presentation decreased, both forward and reversed motion were severely affected, and performance in both conditions dropped close to chance levels. Both observers showed some moderate forward performance at the highest carrier contrasts only, which may have been caused by the intrusion of a distortion product due to the high carrier contrast (Burton, 1973; Derrington, 1994; Henning et al., 1975; Holliday \& Anderson, 1994).

In Experiment 2.2 (Figures 4A-4C), there was some recovery of veridical performance in the $4^{\circ}$ central condition for the high temporal frequency envelope. Very little reversal was perceived in this condition, but as carrier contrast increased, some forward motion was perceived. It has been suggested that this effect may be due to a firstorder artifact introduced into the stimulus prior to initial spatial filtering (Derrington, 1994; Holliday \& Anderson, 1994). If this were the case, one would expect this artifact to behave in a way very similar to that for a real grating at this spatial and temporal frequency. Figure 7B plots direction discrimination performance for a $0.1: 1$-cpd AM luminance grating and a 0.1 -cpd carrier grating presented in a 


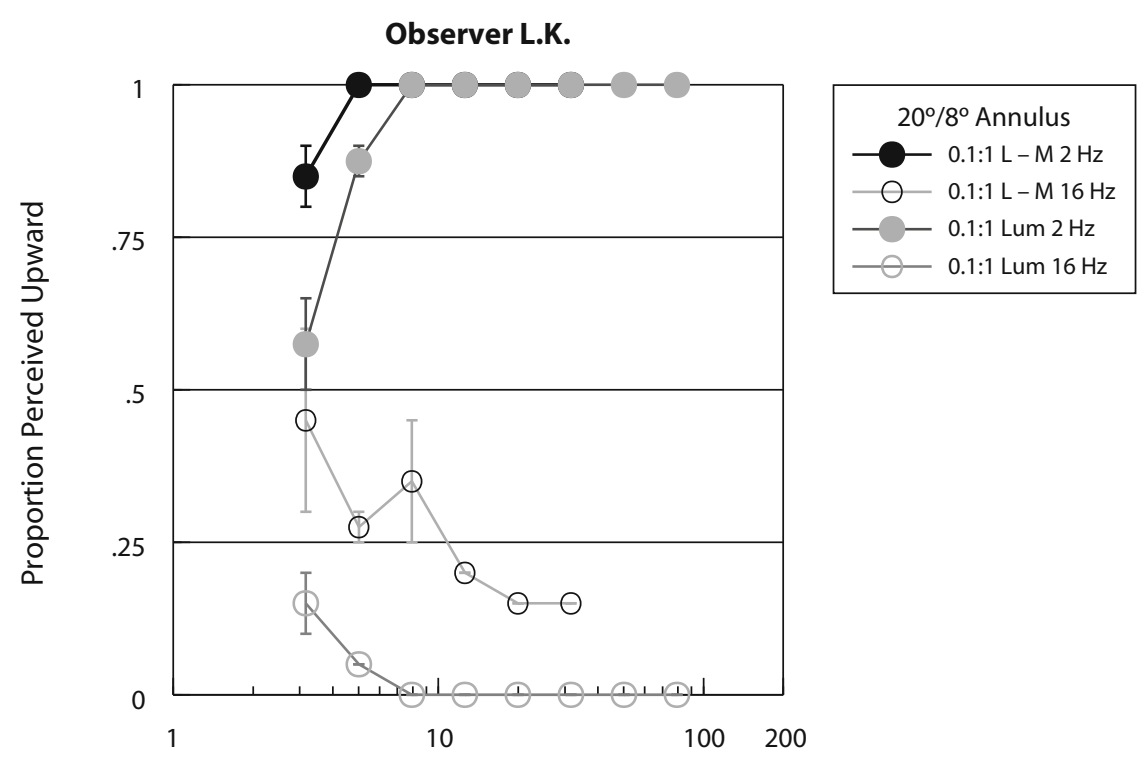

Carrier Contrast (Multiples Above Threshold)

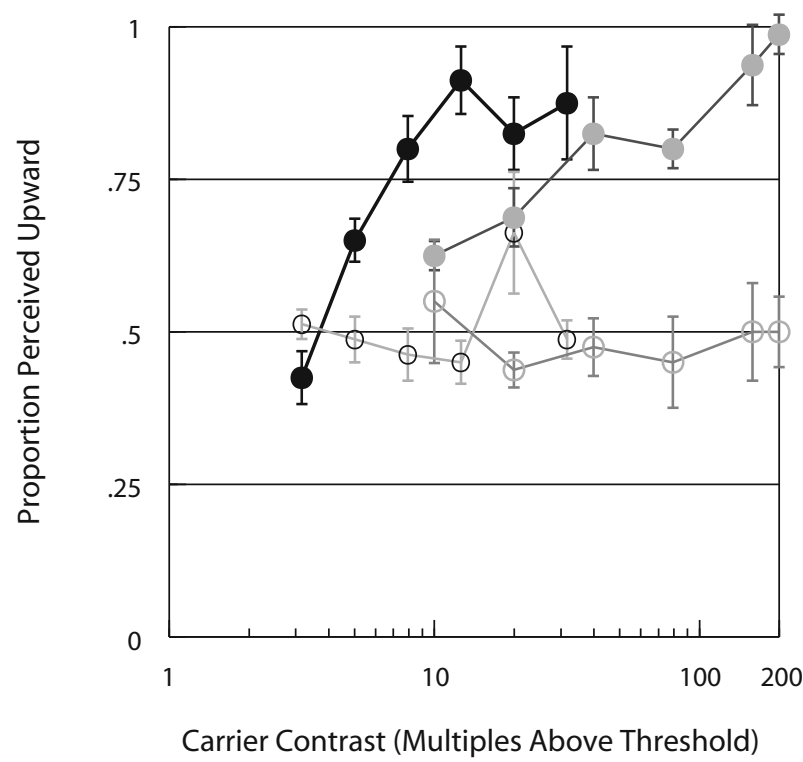

$4^{\circ}$ Central; $50 \%$ Modulation Depth

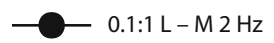

O- $0.1: 1 \mathrm{~L}-\mathrm{M} 16 \mathrm{~Hz}$

- 0.1:1 Lum $2 \mathrm{~Hz}$

- $0.1: 1 \mathrm{Lum} 16 \mathrm{~Hz}$

Figure 6D. Experiment 2.4: Details as in Figure 6C, except that additional conditions of a $20^{\circ} / 8^{\circ}$ annulus and $4^{\circ}: 50 \%$-modulated envelope are added.

$4^{\mathrm{o}}$ central disk as a function of carrier contrast; the symbol size indicates the duration of presentation (the larger symbol indicates the longer duration). As shown in the previous figure $(7 \mathrm{~A})$, reducing the duration had a severe effect upon the performance for the envelope motion discrimination, reducing it to chance. The correct direction of motion of the grating alone $(0.1 \mathrm{cpd})$ was clearly discriminable at a duration of $0.0312 \mathrm{sec}$. This is inconsistent with an argument proposing that the envelope and a real grating behave in the same way at high carrier contrasts, which is, generally speaking, the underlying tenet of any distortion product argument.

\section{DISCUSSION}

The work in this article presents a simple, yet extensive, parametric investigation of the properties of contrastmodulated stimuli when observers are required to discriminate their motion across a range of drift frequencies. The major benefit of this study is that it provides a controlled and consistent description of the properties of the subsystem mediating the discrimination of motion direction with a single stimulus type that, as was argued above, may be considered to be the simplest of all second-order stimuli. These data, in turn, constitute an ideal data set with which 

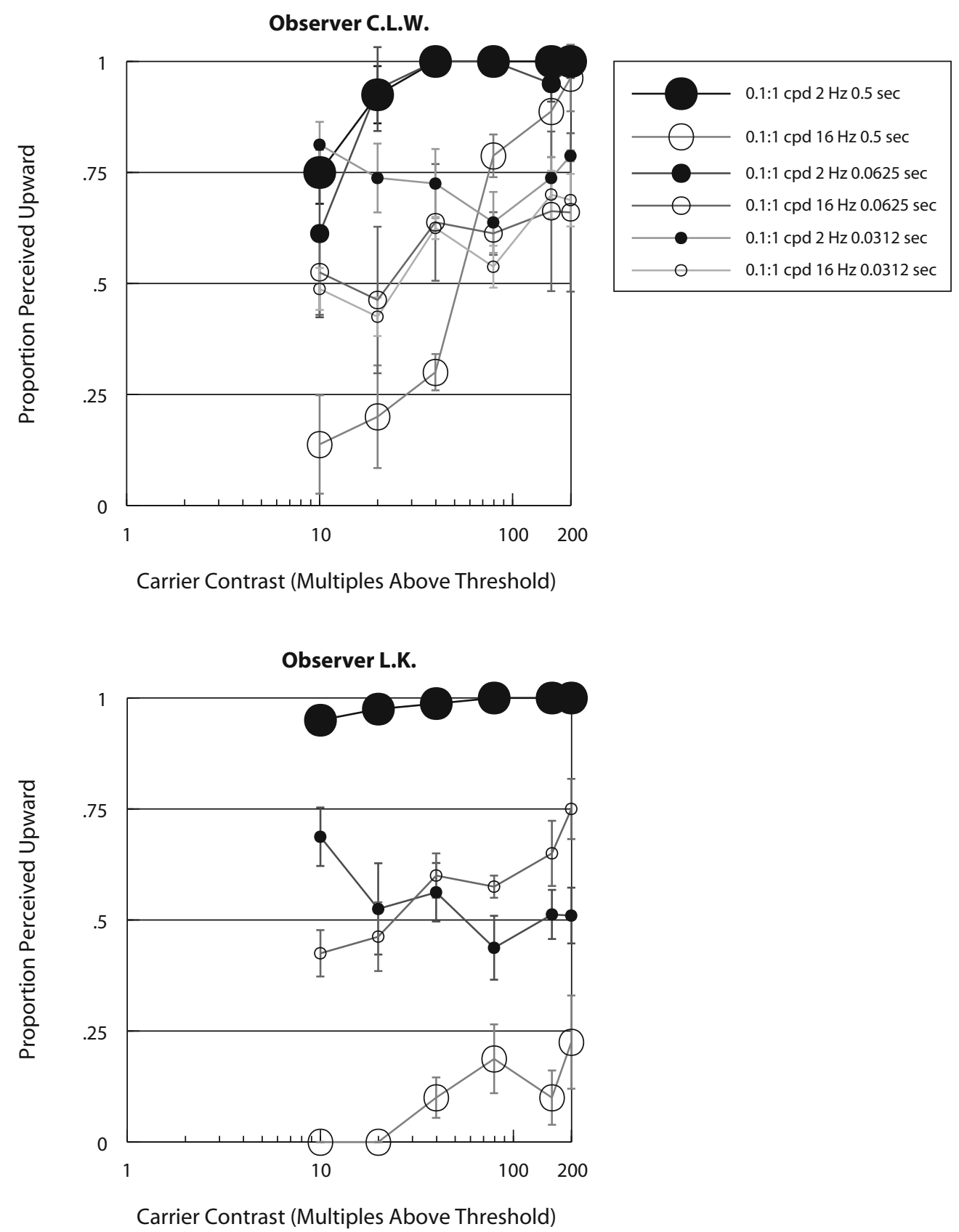

Figure 7A. Experiment 2.5: The effect of stimulus duration on the ability to discriminate amplitudemodulated envelope direction at high and low temporal drift rates in a $20^{\circ} / 8^{\circ}$ annular configuration (to maximize perceived reversal). The proportion correct in the direction discrimination task is plotted against the carrier contrast expressed as multiples of carrier detection threshold for each observer and duration. Carrier $1 \mathrm{cpd}$, envelope $100 \%$ modulated. Three durations are shown $(0.5,0.0625$, and 0.0312 sec) for 2 observers, C.L.W. and L.K. Further details are in the text for Experiment 2.5.

to test models of motion detection purporting to explain our psychophysical ability for all second-order stimuli.

\section{Empirical Summary}

Throughout the study, the temporal drift frequency of the stimulus was held as the independent variable and was used as a yardstick with which to compare direction perception as a function of the variation of other critical stimulus parameters. Particular care was taken to ensure that all patterns were scaled to be equivalent in visibility in terms of both their carrier and their envelope, which facilitates comparison across conditions, stimuli, and observers. The results of Experiment 1 showed an abrupt and decisive perception of reversed motion when the envelope of an AM grating was drifted at a rate greater than around $5 \mathrm{~Hz}$. The manipulation of component spatial frequency 


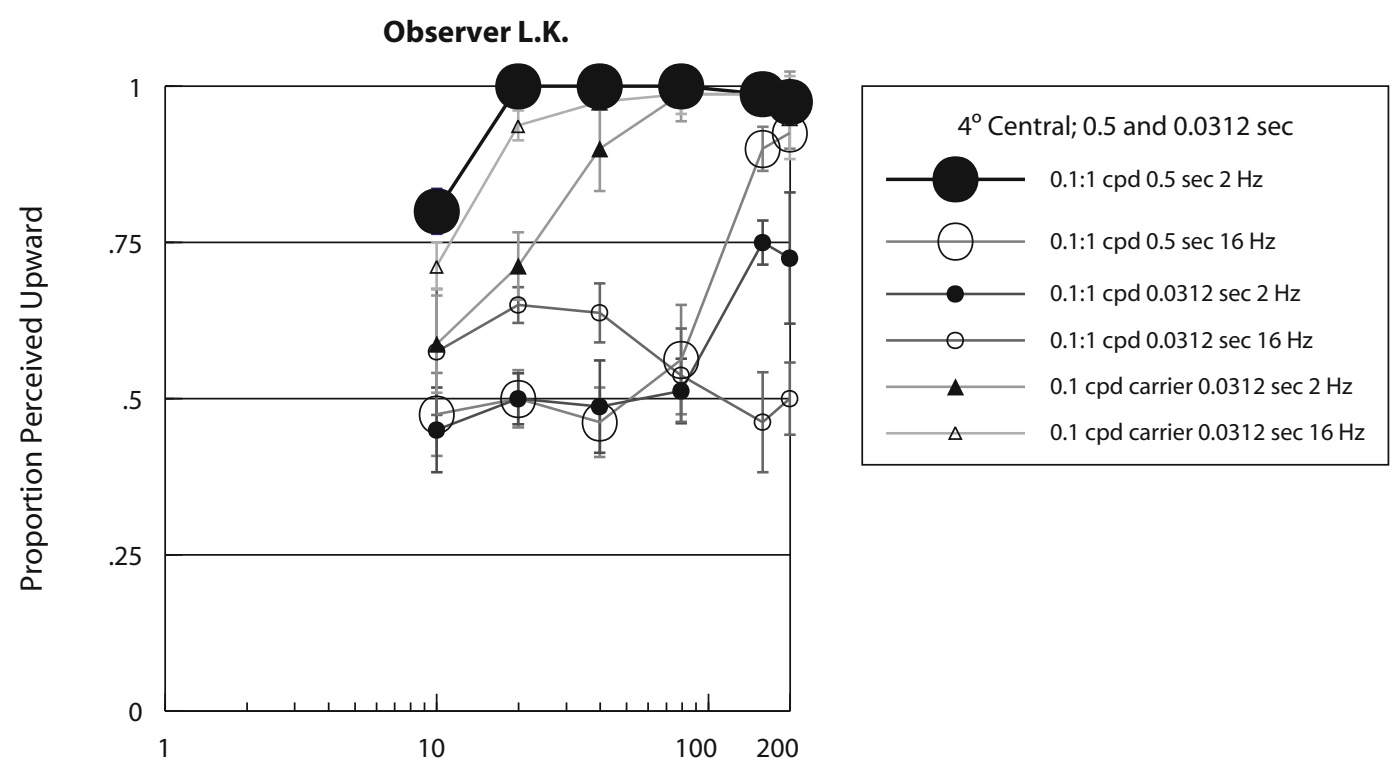

Carrier Contrast (Multiples Above Threshold)

Figure 7B. Experiment 2.5: The effect of stimulus duration for a $4^{\circ}$ central disk, comparing envelope and an equivalent luminance modulation ( $0.1 \mathrm{cpd}$ "carrier" grating). The larger symbols indicate the longer duration of 0.5 sec. Observer, L.K.

(Experiment 2.1), albeit over a restricted range, gave results consistent with the reversal's being strongly related to relative component spatial frequency: The further apart the components were in spatial frequency, the stronger the reversal appeared to be. Changing the location of the stimulus on the retina, in terms of both the overall size and the relative position (Experiments 2.2s and 2.2) also had the predicted effect of increasing the degree of perceived reversal as stimulus placement on the retina moved further into the near periphery. Again, great care was taken that this manipulation was not confounded with stimulus visibility. The change in size of the stimulus highlighted an important effect of local motion on the overall percept, with smaller centrally placed stimuli showing significantly less reversal than did larger or peripheral stimuli under comparable conditions. The precise spatial structure of the stimulus was also considered by comparing AM gratings with beats (only two sideband components: Experiment 2.2s) and QFM gratings (same three components but relativephase-shifted sidebands: Experiment 2.3). Whereas the perception of motion in AM and beat stimuli were shown to be largely similar, as was expected and as had been assumed in much of the previous literature, the reduction in envelope modulation depth inherent in QFM gratings was shown to have a significant effect on forward motion perception in these patterns. Reversal, however, was unaffected, again showing the importance of the magnitude of the components. The less commonly considered stimulus property of carrier color was examined in the penultimate experiment (Experiment 2.4) and was shown to almost completely remove the percept of reversal. Although chromatic and luminance spatiotemporal contrast sensitivity functions are quite different, this was a surprising result, given the careful comparative scaling of the stimuli. Simi- larly, the effect of severely reducing the stimulus duration (Experiment 2.5) did not selectively affect forward motion, as may have been expected. This illustrates that although the components do have a powerful effect on whether a reversal is seen in high-frequency AM stimuli, these components are not treated independently within the system, as if they were separately processed gratings.

\section{Context of the Work}

Recent reviews have highlighted the need for some stock to be taken of the data that we already have on motion detection involving relatively simple stimuli (Cropper \& Wuerger, 2005; Derrington et al., 2004). Before this can be done, we need to be clear on our definitions, and one useful way of defining this "simple" description is to return to the statistical structure of the stimulus. The stimuli used here consisted of a first-order carrier modulated by a secondorder envelope. We manipulated through the stimulus drift frequency the first-order temporal structure of the secondorder spatial modulation (Cropper \& Johnston, 2001). The data presented clarify the temporal properties of any underlying mechanism able to signal the direction of motion of a spatial contrast modulation of color or luminance. In the vast majority of conditions, the maximum temporal sensitivity of such a mechanism was around $4-6 \mathrm{~Hz}$, and a significant reversal was seen around an envelope drift rate of 12-16 Hz. This reversal was not due to a lack of visibility of the stimulus but does seem to have been much stronger for luminance carriers than for chromatic carriers.

\section{The Window of Visibility}

The results can be placed in the context of the window of visibility theory of early visual processing and its relationship to the physical properties of the input stimulus 
(Watson et al., 1986), a fundamental consideration in all psychophysics. This approach has provided a theoretical backdrop in many studies in which an attempt has been made to map out the properties of a given behaviorally defined subsystem of the visual pathway and, as such, is particularly powerful and useful as a first stage in the formalization and modeling of the underlying process. Unfortunately, its importance is often lost when any model of the visual system does not include a spatiotemporal modulation transfer function as its initial stage. The context in which we have placed our data in the present work is to ask whether this initial transfer function - that is, the spatiotemporal contrast sensitivity function of the visual system $^{3}$ - is sufficient to explain the subsequent behavior of the individual in the task at hand (in this case, direction discrimination). It has previously been shown that the window of visibility comprehensively explains our ability to discriminate between smooth motion and sampled motion sequences in chromatic and luminance stimuli but that it does not explain the resultant percept of discontinuous, or jerky, motion in some stimuli (Burr, Ross, \& Morrone, 1986; Cropper \& Badcock, 1994; Mullen \& Boulton, 1992). Thus, the window of visibility can account for some aspects of performance, direction discrimination, while falling short of an explanation of the internal sensation or percept elicited by the stimulus. In line with this, the data presented in the present article strongly imply that the window of visibility does have a powerful influence on the direction of motion seen in these compound patterns but, again, that it does not completely explain the overall percept of forward or reversed motion.

Specifically, changing the component spatial frequency, stimulus location and size, and compound structure of the stimulus, while keeping the component structure constant, all produce data that support the suggestion that the window of visibility qualitatively explains the perceived reversal at high temporal frequencies. On the other hand, the manipulation of stimulus color and duration does not produce data directly in line with this idea, a result that is, oddly, quite consistent with the effects of color and duration in other aspects of motion perception in different patterns (Cropper \& Derrington, 1994; Derrington et al., 1993; Wilson et al., 1992; Yo \& Wilson, 1992).

The cautionary word to note above is that the qualitative support given by the window of visibility explanation falls short of the full quantitative support that we were able to show in previous work on smooth and sampled motion (Cropper \& Badcock, 1994). While considering the window of visibility to be a particularly intuitive and attractive explanation for the data, we need to consider the exact conditions under which our data were collectedthat is, at low spatial frequencies with the principal dimension of manipulation being temporal frequency - in order to quantify the degree to which the data may support this account. Examination of the spatial and temporal contrast sensitivity functions in the regions of interest raises caution in the default, window of visibility, as the sole explanation for the present results (Robson, 1966). Our components were centered on $1 \mathrm{cpd}$ in all cases and varied only in their spatial frequency by, at most, $0.4 \mathrm{cpd}$ and, more often, only $0.2 \mathrm{cpd}$. Thus, any difference in contrast sensitivity to the two components is going to be minor at any temporal frequency. This, in turn, may be why stimulus properties such as location and color appear to be so critical in the appearance of the reversal or otherwise, which suggests that although the reversal may principally be induced by the window of visibility, this is the case only when compounded with other stimulus factors. The stimulus properties of color and duration will clearly have an effect upon the window of visibility itself, but it seems that their impact upon the perceived motion exceeds that which can be predicted simply from a window of visibility explanation. Furthermore, it also seems to be that any slight imbalance in the internal representation of the signal can have a strong effect on the resultant perception of direction and, in the present example, induce a powerful sense of reversal. It is worth noting that the window of visibility - that is, the spatiotemporal contrast sensitivity function - has also been measured for second-order modulation (Cropper, 1998; Hutchinson \& Ledgeway, 2006; Schofield \& Georgeson, 2003), although the second-order window of visibility is itself limited by the sensitivity to the first-order components of the pattern.

\section{Interaction Between Spatial "Channels"}

A recent observation that may be pertinent in this context is that at very short stimulus durations, there is some degree of directional inhibition between similar spatial frequency first-order components in a compound pattern (Serrano-Pedraza, Goddard, \& Derrington, 2007). Although this is described in terms of inhibition between directional mechanisms tuned to the same direction but different spatial frequencies, what the result shows is the existence of unexpected interactions between moving components in a pattern that does not fit into the standard independent-channel approach to early visual processes (Blakemore \& Campbell, 1968, 1969; Campbell \& Robson, 1966). The observation is also closely related to earlier work on the relationship between the grating components of a compound pattern (Derrington \& Goddard, 1989, 1992; Derrington \& Henning, 1987; Henning \& Derrington, 1988). Although each of these studies was concerned with discrimination of the motion of first-order modulation in the pattern and, in the most recent work, the authors took steps to overcome the cue inherent in any second-order modulation (Serrano-Pedraza et al., 2007), there is still some relevance to the present work. This is particularly the case given our proposal that the reversal is caused not by the second-order modulation per se but by the residual motion signals in the stimulus when the second-order-sensitive motion mechanism is insensitive to the increased temporal frequency profile of the stimulus. Both increased drift rate and short stimulus duration bias the temporal frequency profile of the stimulus toward a higher range. If there are interactions between similarly tuned directional mechanisms and there is some opponent arrangement in their respective wiring, it cannot be discounted that there is also some concomitant effect on perceived motion in the opposite direction. This effect may even be to enhance a perceived reversal in some cir- 
cumstances. It is also the case that the perceived direction of motion of the second-order modulation is critically affected by the orientation and spatial frequency components of the first-order carrier, particularly when the pattern is 2-D (Cropper \& Badcock, 2008; Cropper, Badcock, \& Hayes, 1994; Cropper \& Johnston, 2001; Wilson \& Kim, 1994). Both of these additional factors raise the possibility that any nonlinear and unexpected interactions affecting the perceived direction of motion in compound stimuli (Serrano-Pedraza et al., 2007) may also have had some influence on the present results, particularly as the stimulus temporal profile is biased toward higher frequencies.

Interaction between the individual components of the stimuli may also go some way toward explaining why the window of visibility provides only qualitative support for the data. The basic theory, although powerful, does consider only the independent detectability of the components and does not incorporate any intercomponent interaction. Including the consideration that high temporal frequency components interact with one another in nonlinear and unexpected ways allows for the initial qualitative support to be a more satisfying explanation. It certainly circumvents the need to incorporate any less well-supported properties of the system in that explanation, such as additional parallel processes or pathways. It is also the case that the system will make a "decision" when faced with particularly noisy stimuli and that this will affect measured performance, a critical aspect of the more recent work on attention, internal signal strength, and decision making (Grossberg \& Pilly, 2008; Palmer, Huk, \& Shadlen, 2005). This capacity, and necessary requirement, of the system to be able to make some "sense" of a very noisy dynamic neural image of the stimulus not only is critical to consider, but also is a particularly compelling way to overcome the disparity between the psychophysical response (a very strong reversal of perceived motion) and the predicted neural representation of the stimulus (from very little difference in individual component sensitivity). Thus, a very slight difference in sideband representation can have a strong influence on the decision-making process, particularly when the empirical data show that sufficient time to sample the stimulus is also needed to come to this decision (Experiment 2.5).

\section{Conclusions}

The results presented here, in conjunction with those in previous work from the same source (Cropper, 1998; Cropper \& Badcock, 2008; Cropper \& Derrington, 1996; Cropper \& Johnston, 2001), give a clearer picture of the relationship between first- and second-order spatial modulation, the particular spatiotemporal stimulus characteristics, and the ability to discriminate the direction of motion of that modulation, an acid test for any model of human motion perception.

\section{AUTHOR NOTE}

The work presented in this article was collected over a period of several years and was financially supported by grants from the Wellcome Trust and the Australian Research Council. The authors thank Bradley Wolfgang and Chris Benton for insightful comments on the manuscript and the reviewers for their considered comments, and for their stamina! Correspondence concerning this article should be addressed to S. J. Cropper, Department of Psychology, University of Melbourne, Melbourne, Victoria 3010, Australia (e-mail: scropper@unimelb.edu.au).

\section{REFERENCES}

BADCOCK, D. R., \& Derrington, A. M. (1985). Detecting the displacement of periodic patterns. Vision Research, 25, 1253-1258.

BADCock, D. R., \& Derrington, A. M. (1987). Detecting the displacements of spatial beats: A monocular capability. Vision Research, 27, 793-797.

BadCock, D. R., \& Derrington, A. M. (1989). Detecting the displacements of spatial beats: No role for distortion products. Vision Research, 29, 731-739.

BADCOCK, D. R., \& KHUU, S. K. (2001). Independent first- and secondorder motion energy analyses of optic flow. Psychological Research, 65, 50-56.

Benton, C. P., \& Johnston, A. (1997). First-order motion from contrast modulated noise? Vision Research, 37, 3073-3078.

Benton, C. P., \& Johnston, A. (2001). A new approach to analysing texture-defined motion. Proceedings of the Royal Society B, 268, 2435-2443.

Benton, C. P., Johnston, A., \& McOwan, P. W. (1997). Perception of motion direction in luminance- and contrast-defined reversed-phi motion sequences. Vision Research, 37, 2381-2399.

BlaKemore, C., \& CAMpbell, F. W. (1968). Adaptation to spatial stimuli. Journal of Physiology, 200, 11P-13P.

Blakemore, C., \& Campbell, F. W. (1969). On the existence of neurones in the human visual system selectively sensitive to the orientation and size of retinal images. Journal of Physiology, 203, 237-260.

Boulton, J. C., \& BAKer, C. L., JR. (1993). Dependence on stimulus onset asynchrony in apparent motion: Evidence for two mechanisms. Vision Research, 33, 2013-2019.

Burr, D. C., Ross, J., \& Morrone, M. C. (1986). Smooth and sampled motion. Vision Research, 26, 643-652.

BuRTON, G. J. (1973). Evidence for nonlinear response processes in the human visual system from measurements on the thresholds of spatial beat frequencies. Vision Research, 13, 1211-1225.

CAmpbell, F. W., \& Robson, J. G. (1966). Application of Fourier analysis to the visibility of gratings. Journal of Physiology, 197, 551-566.

Cavanagh, P., \& Mather, G. (1989). Motion: The long and short of it. Spatial Vision, 4, 103-129.

Chubb, C., \& Sperling, G. (1988). Drift-balanced random stimuli: A general basis for studying non-Fourier motion perception. Journal of the Optical Society of America A, 5, 1986-2006.

Cropper, S. J. (1992). Human motion detection: Different patterns, different detectors? Unpublished doctoral thesis, University of Newcastle upon Tyne, Newcastle, U.K.

Cropper, S. J. (1994). Velocity discrimination in chromatic gratings and beats. Vision Research, 34, 41-48.

CRopper, S. J. (1998). Detection of chromatic and luminance contrast modulation by the visual system. Journal of the Optical Society of America A, 15, 1969-1986.

Cropper, S. J. (2005). The detection of motion in chromatic stimuli: First-order and second-order spatial structure. Vision Research, 45, 265-280.

CropPER, S. J., \& BADCOCK, D. R. (1994). Discriminating smooth from sampled motion: Chromatic and luminance stimuli. Journal of the Optical Society of America A, 11, 515-530.

Cropper, S. J., \& BADCOCK, D. R. (1995). Perceived direction of motion: It takes all orientations [Abstract]. Perception, 24, 106a.

Cropper, S. J., \& BADCOCK, D. R. (2008). Perception of direction of motion reflects the early integration of first and second-order stimulus spatial properties. Journal of Vision, 8, 1-10.

Cropper, S. J., Badcock, D. R., \& HaYes, A. (1994). On the role of second-order signals in the perceived direction of motion of type II plaid patterns. Vision Research, 34, 2609-2612.

Cropper, S. J., \& Derrington, A. M. (1994). Motion of chromatic stimuli: First-order or second-order? Vision Research, 34, 49-58.

Cropper, S. J., \& Derrington, A. M. (1996). Detection and motion detection in chromatic and luminance beats. Journal of the Optical Society of America A, 13, 401-407. 
Cropper, S. J., \& Johnston, A. (2001). The motion of contrast envelopes: Peace and noise. Journal of the Optical Society of America A, 18, 2237-2254.

Cropper, S. J., Johnston, A., \& Benton, C. (2008). The detection of the motion of contrast modulation: Theoretical considerations. Manuscript in preparation.

Cropper, S. J., \& Wuerger, S. M. (2005). The perception of motion in chromatic stimuli. Behavioural \& Cognitive Neuroscience Reviews, 4, 192-217.

Derrington, A. M. (1994). Analysis of the motion of contrast modulated patterns. Investigative Ophthalmology \& Visual Science, 35, 1406.

Derrington, A. M., Allen, H. A., \& Delicato, L. S. (2004). Visual mechanisms of motion analysis and motion perception. Annual Review of Psychology, 55, 181-205.

Derrington, A. M., Badcock, D. R., \& Henning, G. B. (1993). Discriminating the direction of second-order motion at short stimulus durations. Vision Research, 33, 1785-1794.

Derrington, A. M., \& Cox, M. (1998). Temporal resolution of dichoptic and second-order motion mechanisms. Vision Research, 38, 35313539 .

Derrington, A. M., \& Goddard, P. A. (1989). Failure of motion discrimination at high contrasts: Evidence for saturation. Vision Research, 29, 1767-1776.

Derrington, A. M., \& Goddard, P. A. (1992). Does the human visual system combine information from motion analyses at different spatial scales? Journal of Physiology, 452, 283P.

Derrington, A. M., \& Henning, G. B. (1987). Errors in directionof-motion discrimination with complex stimuli. Vision Research, 27, 61-75.

Derrington, A. M., \& Henning, G. B. (1993). Linear and non-linear mechanisms in pattern vision. Current Biology, 3, 800-803.

Derrington, A. M., Krauskopf, J., \& Lennie, P. (1984). Chromatic mechanisms in lateral geniculate nucleus of macaque. Journal of Physiology, 357, 241-265.

DuFFy, C. J. (2004). The cortical analysis of optic flow. In L. Chalupa \& J. S. Werner (Eds.), The visual neurosciences (Vol. 2, pp. 1260-1283). Cambridge, MA: MIT Press.

EDWARDS, M., \& BADCOCK, D. R. (1995). Global motion perception: No interaction between the first-order and second-order motion pathways. Vision Research, 35, 2589-2602.

FINDLAY, J. M. (1978). Estimates on probability functions: A more virulent PEST. Perception \& Psychophysics, 23, 181-185.

GREEN, D. G. (1970). Regional variations in the visual acuity for interference fringes on the retina. Journal of Physiology, 207, 351-356.

GrossberG, S., \& Pilly, P. K. (2008). Temporal dynamics of decisionmaking during motion perception in the visual cortex. Vision Research, 48, 1345-1373.

Henning, G. B., \& Derrington, A. M. (1988). Direction-of-motion discrimination with complex patterns: Further observations. Journal of the Optical Society of America A, 5, 1759-1766.

Henning, G. B., Hertz, B. G., \& Broadbent, D. E. (1975). Some experiments bearing on the hypothesis that the visual system analyses spatial patterns in independent bands of spatial frequency. Vision Research, 15, 887-897.

Holliday, I. E., \& ANDERSON, S. J. (1994). Different processes underlie the detection of second-order motion at low and high temporal frequencies. Proceedings of the Royal Society B, 257, 165-173.

Hutchinson, C. V., \& Ledgeway, T. (2006). Sensitivity to spatial and temporal modulations of first-order and second-order motion. Vision Research, 46, 324-335.

Jamar, J. H. T., Campagne, J. C., \& Koenderink, J. J. (1982). Detectability of amplitude- and frequency-modulation of suprathreshold sine-wave gratings. Vision Research, 22, 407-416.

Jamar, J. H. T., Kwakman, L. F. T., \& Koenderink, J. J. (1984). The sensitivity of the peripheral visual system to amplitude-modulation and frequency-modulation of sine-wave patterns. Vision Research, 24, 243-249.

Johnston, A., \& Benton, C. P. (1997). Speed discrimination thresholds for first- and second-order bars and edges. Vision Research, 37, 2217-2226.

Johnston, A., Benton, C. P., \& McOwan, P. W. (1999). Induced mo- tion at texture-defined motion boundaries. Proceedings of the Royal Society $B$, 266, 2441-2450.

Johnston, A., Benton, C. P., \& Morgan, M. J. (1999). Concurrent measurement of perceived speed and speed discrimination threshold using the method of single stimuli. Vision Research, 39, 3849-3855.

Johnston, A., \& Clifford, C. W. G. (1995). Perceived motion of contrast-modulated gratings: Predictions of the multi-channel gradient model and the role of full-wave rectification. Vision Research, 35, 1771-1784.

Julesz, B. (1971). Foundations of cyclopean perception. Chicago: University of Chicago Press.

KitagaWa, N., \& ICHigara, S. (2002). Hearing visual motion in depth. Nature, 416, 172-174.

Ledgeway, T., \& Hess, R. F. (2000). The properties of the motiondetecting mechanisms mediating perceived direction in stochastic displays. Vision Research, 40, 3585-3597.

LEDGEWAy, T., \& Hess, R. F. (2002). Failure of direction identification for briefly presented second-order motion stimuli: Evidence for weak direction selectivity of the mechanisms encoding motion. Vision Research, 42, 1739-1758.

Ledgeway, T., Hess, R. F., \& McGraw, P. V. (2002). Masking effects between local first-order and second-order motions in the extraction of global-motion direction depend critically on stimulus visibility. Perception, 31, 99-100.

Ledgeway, T., \& Hutchinson, C. V. (2005). The influence of spatial and temporal noise on the detection of first-order and second-order orientation and motion direction. Vision Research, 45, 2081-2094.

Ledgeway, T., \& Hutchinson, C. V. (2006). Is the direction of secondorder, contrast-defined motion patterns visible to standard motionenergy detectors: A model answer? Vision Research, 46, 556-567.

LedgewAy, T., \& Smith, A. T. (1994a). The duration of the motion aftereffect following adaptation to first-order and second-order motion. Perception, 23, 1211-1219.

Ledgeway, T., \& SMith, A. T. (1994b). Evidence for separate motiondetecting mechanisms for first-order and second-order motion in human vision. Vision Research, 34, 2727-2740.

Ledgeway, T., \& SMith, A. T. (1995). The perceived speed of secondorder motion and its dependence on stimulus contrast. Vision Research, 35, 1421-1434.

Ledgeway, T., \& Smith, A. T. (1997). Changes in perceived speed following adaptation to first-order and second-order motion. Vision Research, 37, 215-225.

Lennie, P., \& Movshon, J. A. (2005). Coding of color and form in geniculostriate pathway. Journal of the Optical Society of America A, 22, 2013-2033.

LeVinson, E., \& SeKuler, R. (1975a). The independence of channels in human vision selective for direction of movement. Journal of Physiology, 250, 347-366.

LEVINSON, E., \& SEKULER, R. (1975b). Inhibition and disinhibition of direction-specific mechanisms in human vision. Nature, 254, 692694.

Lombrozo, T., Judson, J., \& MacLeod, D. I. A. (2005). Flexibility of spatial averaging in visual perception. Proceedings of the Royal Society B, 272, 725-732.

Lu, Z.-L., \& SPERLING, G. (1995). The functional architecture of human visual motion perception. Vision Research, 35, 2697-2722.

Lu, Z.-L., \& SPERLing, G. (2001). Three systems theory of human visual motion perception: Review and update. Journal of the Optical Society of America A, 18, 2331-2370.

MacLeod, D. I. A., Williams, D. R., \& Makous, W. (1992). A visual non-linearity fed by single cones. Vision Research, 32, 347-363.

MARr, D. (1982). Vision: A computational investigation into the human representation and processing of visual information. San Francisco: Freeman.

Marr, D., \& Ullman, S. (1981). Directional selectivity and its use in early visual processing. Proceedings of the Royal Society B, 211, 151180.

Mullen, K. T. (1985). The contrast sensitivity of human color vision to red-green and blue-yellow chromatic gratings. Journal of Physiology, 359, 381-400.

Mullen, K. T., \& Boulton, J. C. (1992). Absence of smooth motion perception in color vision. Vision Research, 32, 483-488. 
Palmer, J., Huk, A. C., \& Shadlen, M. N. (2005). The effect of stimulus strength on the speed and accuracy of a perceptual decision. Journal of Vision, 5, 376-404.

PANTLE, A. (1992). Immobility of some second-order stimuli in human peripheral vision. Journal of the Optical Society of America A, 9, 863-867.

Robson, J. G. (1966). Spatial and temporal contrast sensitivity functions of the visual system. Journal of the Optical Society of America, 56, 1141-1142.

Schofield, A. J., \& Georgeson, M. A. (2003). Sensitivity to contrast modulation: The spatial frequency dependence of second-order vision. Vision Research, 43, 243-259.

Seiffert, A. E., \& Cavanagh, P. (1998). Position displacement, not velocity, is the cue to motion detection of second-order stimuli. Vision Research, 38, 3569-3582.

Serrano-Pedraza, I., Goddard, P., \& Derrington, A. M. (2007). Evidence for reciprocal antagonism between motion sensors tuned to coarse and fine features. Journal of Vision, 7, 1-14.

Smith, A. T., Hess, R. F., \& BAKer, C. L., JR. (1994). Direction identification thresholds for second-order motion in central and peripheral vision. Journal of the Optical Society of America A, 11, 506-514.

Smith, A. T., \& Ledgeway, T. (1995). Second-order motion: The carrier is crucial [Abstract]. Perception, 24, 28a.

Smith, A. T., \& LedGeWAy, T. (1997). Separate detection of moving luminance and contrast modulations: Fact or artifact? Vision Research, $37,45-62$.

Smith, A. T., \& Ledgeway, T. (1998). Sensitivity to second-order motion as a function of temporal frequency and eccentricity. Vision Research, 38, 403-410.

Smith, A. T., \& Ledgeway, T. (2001). Motion detection in human vision: A unifying approach based on energy and features. Proceedings of the Royal Society B, 268, 1889-1899.

Solomon, J. A., \& Sperling, G. (1995). 1st- and 2nd-order motion and texture resolution in central and peripheral vision. Vision Research, 35, 59-64.

Stone, L. S., \& Thompson, P. (1992). Human speed perception is contrast dependent. Vision Research, 32, 1535-1549.

Thibos, L. N., Cheney, F. E., \& WAlsh, D. J. (1987). Retinal limits to the detection and resolution of gratings. Journal of the Optical Society of America A, 4, 1524-1529.

Thompson, P. (1982). Perceived rate of movement depends on contrast. Vision Research, 22, 377-380.

THOMPSON, P. (1984). The coding of velocity of movement in the human visual system. Vision Research, 24, 41-45.

Virsu, V., Rovamo, J., LAurinen, P., \& NäsÄNen, R. (1982). Temporal contrast sensitivity and cortical magnification. Vision Research, 22, 1211-1217.

WANG, Y.-Z., Hess, R. F., \& BAKER, C. L., JR. (1997). Second-order motion perception in peripheral vision: Limits of early filtering. Journal of the Optical Society of America A, 14, 3145-3154.

WARren, W. H. (2004). Optic flow. In L. Chalupa \& J. S. Werner (Eds.), The visual neurosciences (pp. 1247-1259). Cambridge, MA: MIT Press.

Watson, A. B., Ahumada, A., \& Farrell, J. E. (1986). Window of visibility: A psychophysical theory of fidelity in time-sampled visual displays. Journal of the Optical Society of America A, 3, 300-307.

Watson, A. B., Thompson, P. G., Murphy, B. J., \& Nachmias, J. (1980). Summation and discrimination of gratings moving in opposite directions. Vision Research, 20, 341-347.

Wilson, H. R., Ferrera, V. P., \& Yo, C. (1992). A psychophysically motivated model for two-dimensional motion perception. Visual Neuroscience, 9, 79-97.

Wilson, H. R., \& KIM, J. (1994). Perceived motion in the vector-sum direction. Vision Research, 34, 1835-1842.

Yo, C., \& Wilson, H. R. (1992). Perceived direction of moving twodimensional patterns depends on duration, contrast and eccentricity. Vision Research, 32, 135-147.

\section{NOTES}

1. Although the motion reversal examined in the present article is principally an issue with narrow-band carriers, there are some conditions in which a microbalanced noise carrier can induce a perceived reversal at high drift frequencies (Cropper \& Johnston, 2001).

2 . The terminology used in this article will refer to an amplitude modulated grating (Equation 5) specifically as an AM grating and will use the term amplitude modulation to describe generally the variation in the contrast envelope of a given stimulus.

3. By this, we do not mean simply the modulation transfer function of the lens and the eye, but every stage of the system prior to the extraction of a directional signal that limits the stimulus resolution.

\section{SUPPLEMENTAL MATERIALS}

Supplemental data for Experiment 2.2 may be downloaded from app .psychonomic-journals.org/content/supplemental.

(Manuscript received August 14, 2008; revision accepted for publication November 27, 2008.) 